International Institute for Applied Systems Analysis • A-2361 Laxenburg • Austria Tel: +432236807• Fax: +43223671313• E-mail: info@iiasa.ac.at•Web: www.iiasa.ac.at

INTERIM REPORT

IR-97-013 / March

\title{
A Baseline Model of Industry Evolution
}

S.G. Winter (winter@wharton.upenn.edu)

Y. M. Kaniovski (kaniov@iiasa.ac.at)

G. Dosi (dosi@iiasa.ac.at)

\section{Approved by}

Gordon J. Mac Donald (macdon@ilasa .ac .at)

Director, IIASA

Interim Reports on work of the International Institute for Applied Systems Analysis receive only limited review. Views or opinions expressed herein do not necessarily represent those of the Instiute, its National Memeber Organizations, or other organizations supporting the work. 


\section{Summary}

The paper analyses the properties and outcomes of competitive dynamics in industries characterized by heterogeneous firms and continuing stochastic entry. A formal analytical apparatus is developed, able to derive some generic properties of the underlying competition process combining persistent technological heterogeneity, differential growth of individual firms and turnover.

Key words: evolution, competition, stochastic entry, industrial dynamics.

Journal of Economic Literature Classification Numbers: L11, O30, C60. 


\section{Contents}

1 Introduction $\quad 1$

2 The Basic Model 4

3 Two Dynamical Settings $\quad 6$

4 Some Heuristic Observations Concerning Long Run $\begin{array}{ll}\text { Properties } & 10\end{array}$

$5 \quad$ Entry, Mortality and Long Run Balance Relations $\quad 19$

6 Ergodic Properties of the Industry 23

7 Some Results of Computer Simulations $\quad 26$

8 Generalizations of the Model $\quad 31$

9 Conclusions $\quad 32$

$\begin{array}{ll}\text { References } & 33\end{array}$ 


\title{
A Baseline Model of Industry Evolution
}

\author{
S.G. Winter (winter@wharton.upenn.edu) \\ Y. M. Kaniovski (kaniov@iiasa.ac.at) \\ G.Dosi (dosi@iiasa.ac.at)
}

\section{Introduction}

This paper analyses the properties and outcomes of competitive dynamics in industries characterized by heterogeneous firms and continuing stochastic entry. In that setting aggregate economic variables - such as prices, quantities and indirectly distributive shares - are interpreted as stemming from an explicitly dynamic process combining persistent technological heterogeneity, differential growth of individual firms and turnover.

The idea in its essence is certainly not new: indeed it is quite germane to the view of the competitive process held by classical economists and, later, in diverse fashions, by Marshall and Schumpeter. Just think for example of the classical view of prices and profits as attracted by their "normal" level by inflows/outflows of investment, or, conversely, of the famous Marshallian metaphor of industries as "forests" with young, mature and dying trees. However, the static bias of a lot of contemporary work has also meant the neglect of these early dynamic intuitions. This certainly applies to General Equilibrium Analysis, but also to some extent to traditional industrial organizational economics. While it is true that in the "Structure - Conduct - Performance" (SCP) paradigm entry and entry barriers play a prominent role, it is equally true that the analysis, if not entirely static is at least ahistorical: dates and sequences of events have no visible importance ${ }^{1}$.

The empirical phenomena addressed by our model have been examined in several streams of scholarly literature. (A discussion is in Dosi et al. (1997).) There is, first, a substantial body of descriptive work based on longitudinal data sets with large numbers of firms and establishments; particularly prominent among these are the U.S. Census Bureau's Longitudinal Research Database (Dunne, Roberts and Samuelson (1988), Jensen

\footnotetext{
${ }^{1}$ For example, first mover advantages do not appear among the basic determinants of industry structure in the definitive account of the SCP paradigm, namely Scherer and Ross (1990).
} 
and McGuckin (1997)), and a broadly similar data set developed at Statistics Canada (Baldwin (1995)). A great number of specific questions have been answered by these explorations. What stands out impressionistically, however, is the diversity of firms and the sense of continuing, highly dynamic, disequilibrium. The extent of or turnover at the low end of the size distribution if particularly notable. For example, Dunne, Roberts and Samuelson (1988) summarizing the general picture have remarked among other things that "Entry and exit rates at a point in time are ... highly correlated across industries so that industries with higher than average entry rates tend also to have higher than average exit rates." (p. 496) and that "... the market share of each entering cohort generally declines as the cohort ages. This occurs because high exit rates, particularly when the cohort is young, overwhelm any increase in the relative size of the surviving cohort members." (p. $513)$.

Second, in the literature on "industry life cycles" (for overviews, cf. Klepper (1997) and Afuah and Utterback (1997)), the principal focus is the unfolding pattern of industrial evolution over time. Industries and/or product markets are viewed as entities that have historical starting points, that often have broad patterns of development and ultimately disappear. Levels of entry and exit, degrees of concentration and other phenomena are shown to vary systematically within the historical time-frame of the industry's development.

A third relevant literature is that of the "population ecology of organizations" (Hannan and Freeman (1989), Carroll and Hannan (1995), Carroll (1997)). Empirical work in the field is centrally concerned with explaining the variation over time in the number of organizations undertaking a particular type of activity.

The model that follows is meant to explore some generic properties of the processes of industrial evolution, highlighted - of course with much more historical richness - in the foregoing literature. It is a "baseline" model in two different senses.

First, a number of important issues are resolved here by quite simple assumptions. This partly reflects the fact that the paper is in the evolutionary economics tradition, which generally abjures certain kinds of complexity (cf. Nelson and Winter (1982)). For example, imputation to individual actors of high levels of foresight and knowledge of system structure is avoided when simpler alternatives are adequate to explain aggregate phenomena, and there exists no direct empirical support for the more complex assumptions. This approach stands in sharp contrast to more mainstream economic models of 
competition among heterogeneous actors (cf. Ericson and Pakes (1995), Jovanovic (1982), Hopenheyn (1992)), which accept full ex ante rationality of the individual actors as a fundamental modeling constraint. In our view, added rationality is added complication, and the model presented here provides a baseline that will permit an assessment of the incremental explanatory gain from such complications.

Second, we do expect, however, that some of the simple assumptions will require elaboration and modification in future work if the model is to be brought into reasonable correspondence with reality. Hence, the model is a baseline not merely in the sense of a standard for comparison, but also as a starting point for future work. We anticipate that many of the results developed here will have at least heuristic value, if not direct application, in such future work.

In particular, in the following we study the properties of that special case of evolutionary dynamics whereby technological heterogeneity is bound from the start to some fixed menu of efficiency levels. An obvious future extension, straight in the evolutionary spirit, would be to allow for an "open-ended" dynamics whereby both entrants and incumbents continuously learn and discover along the way novel techniques ${ }^{2}$.

After presenting the basics of the model in Section 2, we develop in Section 3 two dynamic settings, namely a first one which analyses industrial dynamics from the point of view of the dynamics of productive capacity associated with different efficiency levels, and a second one which, on the contrary, follows the fate of all individual firms which appear throughout the whole dynamics path.

An important question is of course whether the purported evolutionary dynamic leads to some "point of rest", i.e. whether there is a deterministic limit. In Section 4, we heuristically discuss this question. While some rest points can be identified under rather awkward assumptions (including the absence of mortality), this cannot be proved in general.

Our model entails a stochastic system driven by the persistent random arrival of new firms, on the one hand, and on a systematic selection process linking investments (and ultimately survival) to realized profitabilities, on the other. Some properties of this system are analyzed in Sections 5 and 6, with respect to its "laws of motion" and the timeaverages of aggregate statistics such as the productive capacities and the numbers of firms in business associated with different efficiency levels.

\footnotetext{
${ }^{2} \mathrm{~A}$ model in this perspective, albeit explored only with simulation techniques is in Dosi, Marsili, Orsenigo and Salvatore (1995).
} 
These analytical results are followed in Section 7 by a computer simulation of the model, showing among other things the dynamics in the number, size and age of firms. Finally, in Section 8 we suggest some easy generalizations which can be treated with the technique duggested here and would not affect the main qualitative conclusions concerning the model.

\section{The Basic Model}

Consider an industry evolving in discrete time $t=0,1, \ldots$. At time $t=0$ there are no firms ready to manufacture, but a random number of firms are drawn which will start manufacturing at $t=1$. At time $t \geq 1$ the industry consists of $n_{t}$ firms which are involved in manufacturing and new firms which enter at $t$ and will be involved in manufacturing from $t+1$ on. Uniformly for the whole industry we have:

$m$ - variable costs per unit of output, $m>0$,

$v$ - price per unit of physical capital, $v>0$,

$d$ - depreciation rate, $0<d \leq 1$.

Competitiveness of a firm represented in the industry is determined by its capital per unit of output ${ }^{3}$ Let us designate it by $a_{i}$ for the $i$-th firm. The variable $a_{i}$ takes a finite number of values $A_{1}<A_{2}<\ldots<A_{k}, k \geq 1$. A particular value is randomly assigned to a firm when it enters the industry.

The productive capacity of the $i$-th firm is $Q_{t}^{i}=K_{i}(t) / a_{i}$, where $K_{i}(t)$ stands for the capital of the $i$-th firm at time $t$. The total productive capacity of the industry involved in manufacturing at time $t$ is

$$
Q_{t}=\sum_{i=1}^{n_{t}} Q_{t}^{i}
$$

(We set that the sum where the lower index exceeds the upper one equals to zero.) There is a decreasing continuous demand function $p=h(q)$, mapping $[0, \infty)$ in $[0, h(0)]$ such that $h(0)<\infty$ and $h(q) \rightarrow 0$ as $q \rightarrow \infty$. Here $p$ stands for the price and $q$ for the demand. Thus, the price at time $t$ is given as $h\left(Q_{t}\right)$. The gross profit per unit of output at $t$ is $h\left(Q_{t}\right)-m$. Hence, the gross investment per unit of output at $t$ reads $\lambda \max \left[h\left(Q_{t}\right)-m, 0\right]$, where $0<\lambda \leq 1$. The constant $\lambda$ captures the share of the gross profit which does not leak out as the interest payments and shareholders' dividends. It can be considered as a

\footnotetext{
${ }^{3}$ Note that the same qualitative results would apply if one made the symmetric opposite assumption of uniform capital/output ratios and heterogeneous variable costs or both (cf. also Section 8).
} 
measure for the propensity to invest. Then the total gross investment per unit of capital for the $i$-th firm at time $t$ is $I\left(Q_{t}\right) Q_{t}^{i} / a_{i}$, where for $x \geq 0$

$$
I(x)=\frac{\lambda}{v} \max [h(x)-m, 0] \geq 0 .
$$

We shall allow for the industry multiple entrants. Capitals of new entrants take values from the interval $[b, c], 0<b<c<\infty$. A particular value is randomly assigned to a firm when it enters the industry. We postulate that the initial capitals are independent realizations of a random variable $\theta$ distributed over $[b, c]$.

At each time $t$ we shall allow a random number of entrants for each level of capital per unit of output. This number is given by an independent realization of a random variable $\gamma(E \gamma \neq \gamma)$ taking the values $0,1, \ldots, l$, where $l$ is a positive integer $(P\{\gamma=l\}>0)$. The number of entrants at time $t$ that have the $j$-th level of capital per unit of output is given by the $j$-th coordinate $\Gamma_{j}^{t}$ of a $k$-dimensional random vector $\Gamma^{t}$. The vectors $\Gamma^{t}$ are realizations of $\boldsymbol{\Gamma}$ independent in $t$. Each coordinate of $\boldsymbol{\Gamma}$ is an independent realization of $\gamma$. The initial capitals of new entrants at time $t$ with the $j$-th level of capital per unit of output are given by $\Gamma_{j}^{t}$ independent realizations $\theta_{j, i}^{t}, 1 \leq i \leq \Gamma_{j}^{t}$, of $\theta$ if $\Gamma_{j}^{t}>0$ and are equal to 0 if $\Gamma_{j}^{t}=0$.

Set $\boldsymbol{\Theta}^{t}$ to be a $k$-dimensional vector such that

$$
\Theta_{j}^{t}=\sum_{i=1}^{\Gamma_{j}^{t}} \theta_{j, i}^{t} .
$$

Thus, $\Theta_{j}^{t}$ represents the total inflow of capital at $t$ of firms with $A_{j}$ as capital per unit of output. The random variables $\Gamma_{j}^{t}$ and $\theta_{p, i}^{s}$ are independent for all possible combinations of indexes. Note that in this model we treat the stochastic entry process as entirely exogenous - in particular entry does not depend on past or present industry profitability. The point of this assumption is not the affirmative claim that all entry is independent of profitability, but that some entry is (especially in the vicinity of equilibrium). Many models of rational entry under uncertainty (to say nothing of models of optimistic bias in entry decisions ${ }^{4}$ ), would produce that result. The principal qualitative result of our analysis would not be affected by the addition of a layer of profit-dependent entry, though the qualitative results certainly is (see also Sections 7 and 8 below).

To accomplish the description of this competitive environment we need some death mechanism. A firm is dead at time $t$ and does not participate in the production process

\footnotetext{
${ }^{4}$ More on it in Dosi and Lovallo (1997).
} 
from $t+1$ on if its capital at $t$ is less than $\epsilon b, \epsilon \in(0,1]$. The situation without mortality can be thought of as a limit case of this threshold when $\epsilon=0$.

We assume that all random elements are given on a probability space $\{\Omega, \mathcal{F}, P\}$.

In the following we shall study the long run behavior of this industry, beginning with a formal description of its evolution.

\section{Two Dynamical Settings}

Set $Q_{1}^{A_{j}}=A_{j}^{-1} \Theta_{j}^{0}$ for the total productive capacity of those firms having $A_{j}$ as capital per unit of output which are involved in manufacturing in the first time period, $j=1,2, \ldots, k$. These firms perform the first cycle of production and new firms come in the industry. As the result, by the end of the first time period the productive capacity $q_{1}^{A_{j}}$ of firms having $A_{j}$ as capital per unit of output reads

$$
q_{1}^{A_{j}}=Q_{1}^{A_{j}}\left[1-d+I\left(Q_{1}\right) A_{j}^{-1}\right]+V_{1}^{j}, \quad j=1,2, \ldots, k,
$$

where

$$
Q_{1}=\sum_{j=1}^{k} Q_{1}^{A_{j}}, \quad V_{1}^{j}=A_{j}^{-1} \Theta_{j}^{1} .
$$

Conceptually, $V_{1}^{j}$ is the total inflow of productive capacity of firms having $A_{j}$ as capital per unit of output during the first time period. $Q_{1}$ stands for the total productive capacity involved in manufacturing during the first time period. Not all of the firms which manufactured during the first production cycle remain in the industry during the second time period. Those that have shrunk below the minimum threshold have to leave. Designate by $\mathcal{E}_{1}^{j} \geq 0$ the total outflow of productive capacity of firms having $A_{j}$ as capital per unit of outputt. Then the total productive capacity $Q_{2}^{A_{j}}$ of firms which are ready to produce during the second time period reads

$$
Q_{2}^{A_{j}}=q_{1}^{A_{j}}-\mathcal{E}_{1}^{j}=Q_{1}^{A_{j}}\left[1-d+I\left(Q_{1}\right) A_{j}^{-1}\right]+V_{1}^{j}-\mathcal{E}_{1}^{j}, \quad j=1,2, \ldots, k .
$$

In the same way we get

$$
Q_{t+1}^{A_{j}}=Q_{t}^{A_{j}}\left[1-d+I\left(Q_{t}\right) A_{j}^{-1}\right]+V_{t}^{j}-\mathcal{E}_{t}^{j}, \quad t \geq 1, \quad j=1,2, \ldots, k .
$$

Here $Q_{t}^{A_{j}}$ stands for the total productive capacity of those firms having $A_{j}$ as capital per unit of output which manufacture during $t$-th production cycle. Also, $V_{t}^{j}$ designates the total inflow of productive capacity of firms having $A_{j}$ as capital per unit of output at time 
$t$, that is, $V_{t}^{j}=A_{j}^{-1} \Theta_{j}^{t}$, and $\mathcal{E}_{t}^{j}$ stands for the total outflow of productive capacity of such firms at time $t$ due to mortality. By $Q_{t}$ we designate the total productive capacity of firms involved in manufacturing at time $t$, that is,

$$
Q_{t}=\sum_{j=1}^{k} Q_{t}^{A_{j}}, \quad t \geq 1
$$

Taking into account (1), we see that this value evolves as

$$
Q_{t+1}=Q_{t}(1-d)+I\left(Q_{t}\right) \sum_{j=1}^{k} A_{j}^{-1} Q_{t}^{A_{j}}+V_{t}-\mathcal{E}_{t}, \quad t \geq 1,
$$

where $V_{t}$ designates the total inflow of productive capacity at time $t$, that is,

$$
V_{t}=\sum_{j=1}^{k} V_{t}^{j}
$$

and $\mathcal{E}_{t}$ stands for the total outflow of productive capacity at time $t$ due to mortality, that is,

$$
\mathcal{E}_{t}=\sum_{j=1}^{k} \mathcal{E}_{t}^{j}
$$

The random process given by (1) and (2) is not a Markov process. However, it turns out to be one, if there is no death rule (and, hence, firms may shrink indefinitely but do not exit the industry).

Note that this setting does not account for the fate of an individual firm. Let us consider an alternative, explicitly microfounded, representation.

Since only the entry process of the model is stochastic, the state of the industry at any time $t$ is determined given the detailed entry and output history through $t-1$ and the stochastic events of $t$. Further, the outputt history of the system to any $t$ can be computed recursively on the basis of prior output history and current stochastic entry. Although only finitely many firm output levels are relevant up to any particular $t$, a full realization of the process involves an infinite number of firm histories. At any time, the part of the outputt history that has not happened yet is represented by an infinite list of zeroes; zeroes may also appear in the firm-specific output history because the corresponding firm has died. It is convenient for the representation to make room for every possible firm that could come into being; this means that zeroes also appear in the output history of a given time because less than the maximum possible number $(l)$ of firms entered in some previous period. 
With this motivation in mind, introduce an infinite dimensional space $R^{\infty}$ of vectors with denumerably many coordinates. Set

$$
R^{\infty}=\bigotimes_{i=1}^{\infty} R_{i}^{k l}
$$

where $\otimes$ stands for the direct sum of $k l$-dimensional real vector spaces $R_{i}^{k l}$. Thus, for every $\mathbf{q} \in R^{\infty}$

$$
\mathbf{q}=\bigotimes_{i=1}^{\infty} \mathbf{q}^{i}
$$

with $\mathbf{q}^{i} \in R_{i}^{k l}$. That is, the infinite outputt history $\mathbf{q}$ may be regarded as partitioned into vectors $\mathbf{q}^{i}$ of dimension $k l$, each of which may be be thought of as output levels of a specific age cohort, $i$ - where, as noted above, we "make room" in the notation for the outputs of firms that may not exist in a particular realization because less than the maximum possible number of entrants appeared in that cohort. The notational convention adopted is that firms are numbered within types, and then arbitrarily within cohort. Thus, for example, the firms of the third cohort are numbered from $2 l+1$ to $3 l$. In a realization of the process, the deterministic part of the output change from period to period can be represented as follows. As a first step, we define an automorphism $\mathbf{D}(\cdot)$ on $R^{\infty}$ such that

$$
\mathbf{D}(\mathbf{q})=\left(\begin{array}{c}
\mathbf{0}^{1} \\
\bigotimes_{i=1}^{\infty} \mathbf{D}^{i}\left(\mathbf{q}^{i}\right)
\end{array}\right),
$$

where $\mathbf{0}^{1}$ stands for the zero vector in $R_{1}^{k l}$. Also

$$
\mathbf{D}^{i}(\cdot): R^{\infty} \mapsto R_{i+1}^{k l}, i \geq 1
$$

Let for $1 \leq j \leq k, \quad 1 \leq p \leq l$, and $i \geq 1$

$$
D_{(j-1) l+p}^{i}\left(\mathbf{q}^{i}\right)=q_{(j-1) l+p}^{i}\left[1-d+I\left(\sum_{i=1}^{\infty} \sum_{s=1}^{k l} q_{s}^{i}\right) A_{j}^{-1}\right] \chi_{L_{(j-1) l+p}^{i}(\mathbf{q})} .
$$

Here $D_{s}^{i}(\cdot)$ stands for the $s$-th coordinate of $\mathbf{D}^{i}(\cdot)$. We restrict ourselves to vectors with nonnegative coordinates and set $I(\infty)=0$ for the case when the iterated sum involved in the above expression is infinite. $L_{(j-1) l+p}^{i}(\mathbf{q})$ stands for the relation

$$
q_{(j-1) l+p}^{i}\left[1-d+I\left(\sum_{i=1}^{\infty} \sum_{s=1}^{k l} q_{s}^{i}\right) A_{j}^{-1}\right] A_{j} \geq \epsilon b
$$

that is, the condition under which the firm whose productive capacity at time $i$ is $q_{(j-1) l+p}^{i}$ survives to period $i+1$, given that at $i$ the productive capacities involved in manufacturing 
are described by the vector $\mathbf{q} \in R^{\infty} \cdot \chi_{L_{(j-1) l+p}^{i}(\mathbf{q})}$ is the indicator function of the relation $L_{(j-1) l+p}^{i}(\mathbf{q})$. We set that for a relation $\mathcal{A}$

$$
\chi_{\mathcal{A}}= \begin{cases}1, & \text { if } \mathcal{A} \text { is true } \\ 0, & \text { otherwise }\end{cases}
$$

The indicator function involved in the definition of $D_{(j-1) l+p}^{i}(\cdot)$ serves for the following purpose. Consider at time (age) $i \geq 1$ an alive firm having $A_{j}$ as capital per unit of output. Let its productive capacity be $q_{(j-1) l+p}^{i}>0$ (since it is alive). The question is whether it will be participating in the next production cycle or not. According to our mortality rule, it depends upon whether its capital at the end of the current production period is not less than or falls below the death threshold, $\epsilon b$. The investment rule adopted in the model gives

$$
q_{(j-1) l+p}^{i}\left[1-d+I\left(\sum_{i=1}^{\infty} \sum_{s=1}^{k l} q_{s}^{i}\right) A_{j}^{-1}\right]
$$

for its production capacity at the end of the current production cycle, or, in capital terms,

$$
q_{(j-1) l+p}^{i}\left[1-d+I\left(\sum_{i=1}^{\infty} \sum_{s=1}^{k l} q_{s}^{i}\right) A_{j}^{-1}\right] A_{j} .
$$

Hence, this firms survives and continues its production if this expression is not less than $\epsilon b$. Otherwise, if it falls below $\epsilon b$, the firm dies and never returns to business.

Define infinite dimensional random vectors $\mathbf{Y}^{t}, t \geq 0$, independent in $t$. Set

$$
Y_{(j-1) l+p}^{t}=\theta_{j, p}^{t} A_{j}^{-1} \text { for } p=1,2, \ldots, \Gamma_{j}^{t}, \quad Y_{(j-1) l+\Gamma_{j}^{t}+i}^{t}=0 \text { for } i=1,2, \ldots, l-\Gamma_{j}^{t}
$$

if $\Gamma_{j}^{t}>0$ and

$$
Y_{(j-1) l+p}^{t}=0 \text { for } p=1,2, \ldots, l
$$

if $\Gamma_{j}^{t}=0$, also

$$
Y_{s}^{t}=0 \text { for } s>k l .
$$

Here $j=1,2, \ldots, k$. Now the evolution of the economy can be given as follows

$$
\mathbf{q}(t+1)=\mathbf{D}(\mathbf{q}(t))+\mathbf{Y}^{t+1}, t \geq 0, \mathbf{q}(0)=\mathbf{Y}^{0}
$$

Since $\mathbf{Y}^{t}$ are independent in $t$, this expression defines a Markov process on $R^{\infty}$. Also, since the deterministic operator $\mathbf{D}(\cdot)$ as well as the distribution of $\mathbf{Y}^{t}$ do not depend on time, the process is homogeneous in time. Conceptually this phase space is formed by productive capacities of all firms which stay alive. More precisely, if $q_{(i-1) k l+(j-1) l+p}(t)>0$ for some 
$p=1,2, \ldots, l$ and $t>i \geq 1$, then a firm with $A_{j}$ as capital per unit of output which came to the industry at $t-i$ has been alive until $t$, that is, has manufactured $i-1$ times, and continue to manufacture during the $t$-th time period.

Having outlined the specific features of this process of industrial change, let us proceed to its analysis.

\section{Some Heuristic Observations Concerning Long Run Properties}

In order to get some intuition on the evolution of this dynamic system, let us start from some heuristic exercises concerning its possible long run behavior. We can consider equation (3) as a stochastic perturbation of the following deterministic difference equation

$$
\mathbf{x}(t+1)=\mathbf{F}(\mathbf{x}(t)), \quad t \geq 0,
$$

where $\mathbf{F}(\mathbf{x})=\mathbf{D}(\mathbf{x})+E \mathbf{Y}^{t}$ for every $\mathbf{x} \in R^{\infty}$. There is no guarantee that the sequence generated by (4) has a limit. But if for some $\mathbf{x}(0)$ it does, the limit $\tilde{\mathbf{x}}$ must be a fixed point with nonnegative coordinates of the mapping $\mathbf{F}(\cdot)$, that is, $\tilde{\mathbf{x}}=\mathbf{F}(\tilde{\mathbf{x}})$ and $\tilde{x}_{i} \geq 0, \quad i \geq 1$. In terms of our model of industrial growth, it would imply that if the process $\mathbf{q}(t), t \geq 1$, concentrates as $t \rightarrow \infty$ close to some deterministic vector, then it can be only $\tilde{\mathbf{x}}$.

Consider the following equation

$$
\mathbf{x}=\mathbf{F}(\mathbf{x})
$$

Note that first $k l$ coordinates of $\mathbf{F}(\cdot)$ are always those of $E \mathbf{Y}^{t}$. Hence,

$$
\tilde{x}_{i}=E Y_{i}^{t}, \quad i=1,2, \ldots, k l,
$$

where

$$
E Y_{l(j-1)+p}^{t}=A_{j}^{-1} E \theta \sum_{i=p}^{l} p_{i}, \quad p=1,2, \ldots, l, \quad p_{i}=P\{\gamma=i\}, \quad i=0,1, \ldots, l .
$$

Also, taking into account the structure of $\mathbf{D}(\cdot)$, we see that

$$
\begin{aligned}
& \tilde{x}_{s k l+l(j-1)+p}=\tilde{x}_{(s-1) k l+l(j-1)+p}[1-d+ \\
& \left.I\left(\sum_{i=1}^{\infty} \tilde{x}_{i}\right) A_{j}^{-1}\right] \chi_{L_{(j-1) l+p}^{s}(\tilde{\mathbf{x}})}, \quad s \geq 1, \quad 1 \leq j \leq k, \quad 1 \leq p \leq l,
\end{aligned}
$$


where $L_{(j-1) l+p}^{s}(\tilde{\mathbf{x}})$ stands for the relation

$$
\tilde{x}_{(s-1) k l+l(j-1)+p}\left[1-d+I\left(\sum_{i=1}^{\infty} \tilde{x}_{i}\right) A_{j}^{-1}\right] A_{j} \geq \epsilon b .
$$

Expressions (6) and (7) allow for the following result.

Lemma 1. If there is a solution $\tilde{\mathbf{x}}$ of equation (5), then its nonzero coordinates are exponentially declining in the number of cohort, that is, if $\tilde{x}_{(s-1) k l+l(j-1)+p}>0$ and $\tilde{x}_{s k l+l(j-1)+p}>0$ for some $s \geq 1$, then

$$
\frac{\tilde{x}_{s k l+l(j-1)+p}}{\tilde{x}_{(s-1) k l+l(j-1)+p}}=1-d+I\left(\sum_{i=1}^{\infty} \tilde{x}_{i}\right) A_{j}^{-1}<1
$$

for all possible $j$ and $p$.

Proof. Since

$$
1-d+I\left(\sum_{i=1}^{\infty} \tilde{x}_{i}\right) A_{1}^{-1}<1
$$

implies

$$
1-d+I\left(\sum_{i=1}^{\infty} \tilde{x}_{i}\right) A_{j}^{-1}<1, \quad j=2,3, \ldots, k,
$$

it is enough to show that inequality (8) holds.

Assume to the contrary that

$$
1-d+I\left(\sum_{i=1}^{\infty} \tilde{x}_{i}\right) A_{1}^{-1} \geq 1 .
$$

Then by (6) and (7) we conclude that

$$
\sum_{i=1}^{\infty} \tilde{x}_{i}=\infty
$$

Hence,

$$
I\left(\sum_{i=1}^{\infty} \tilde{x}_{i}\right)=0,
$$

which implies that

$$
1-d+I\left(\sum_{i=1}^{\infty} \tilde{x}_{i}\right) A_{1}^{-1}=1-d<1 .
$$

Thus, relation (9) leads to a contradiction. Hence, the opposite inequality (8) must hold.

The lemma is proved.

Thus, if $\mathbf{q}(t)$ concentrates as $t \rightarrow \infty$ close to some deterministic vector, then the limit structure of the industry involves infinitely many of infinitely small firms if $\epsilon=0$ and a finite number of firms whose capitals are not less than $\epsilon b$ if $\epsilon>0$. Thus, for $\epsilon=0$ firms 
live infinitely long, while for $\epsilon>0$ each firm lives only a finite time. Since productive capacities of the most efficient firms decline in the number of cohort at the slowest rate, they survive (produce) for the longest time.

Another result which follows from (6) - (8) is the following one.

Lemma 2. Assume that capitals of newcoming firms vanish, that is, for some positive constants $b^{\prime}$ and $c^{\prime}$ we have that $b=\Delta b^{\prime}$ and $c=\Delta c^{\prime}$ with $\Delta \rightarrow 0$. If there is a solution $\tilde{\mathbf{x}}$ of (5), then its nonzero elements vanishes as $\Delta \rightarrow 0$. Furthermore, set

$$
\tilde{X}^{j}=\sum_{i=1}^{\infty} \sum_{s=(i-1) k l+(j-1) l+1}^{i k l+j l} \tilde{x}_{s}, j=1,2, \ldots, k,
$$

for the total, equilibrium, productive capacity of all firms having $A_{j}$ as capital per unit of output. Then as $\Delta \rightarrow 0$

$$
\max _{j=2,3, \ldots, k} \tilde{X}^{j} \rightarrow 0
$$

and

$$
\tilde{X}^{1} \rightarrow Q^{A_{1}}
$$

where $Q^{A_{j}}=h^{-1}\left(m+d v A_{j} / \lambda\right)$ with $h^{-1}(\cdot)$ designating the inverse function.

Proof. Since

$$
1-d+I\left(\sum_{i=1}^{\infty} \tilde{x}_{i}\right) A_{1}^{-1}<1,
$$

by monotonicity of $h^{-1}(\cdot)$ we conclude that

$$
\sum_{i=1}^{\infty} \tilde{x}_{i}>Q^{A_{1}}
$$

For the same reason we see that for $j=2,3, \ldots, k$ and $s \geq 1$

$$
\tilde{x}_{s k l+l(j-1)+p}<\left[1-d+I\left(Q^{A_{1}}\right) A_{j}^{-1}\right] \tilde{x}_{(s-1) k l+l(j-1)+p}, \quad p=1,2, \ldots, l .
$$

Hence, by (6) and (7),

$$
\tilde{X}^{j} \leq \frac{A_{j}^{-1} E \theta E \gamma}{d-I\left(Q^{A_{j}}\right)}, \quad j=2,3, \ldots, k .
$$

Now, since $E \theta<\Delta c^{\prime} \rightarrow 0$ as $\Delta \rightarrow 0$, we obtain relations (10).

We have showed that

$$
\sum_{j=1}^{k} \tilde{X}^{j}>Q^{A_{1}} \quad \text { and } \quad \sum_{j=2}^{k} \tilde{X}^{j} \rightarrow 0 \quad \text { as } \Delta \rightarrow 0,
$$


hence,

$$
\liminf _{\Delta \rightarrow 0} \tilde{X}^{1} \geq Q^{A_{1}}
$$

By (7) we get that

$$
\tilde{X}^{1} \leq \tilde{X}^{1}\left[1-d+I\left(\sum_{j=1}^{k} \tilde{X}^{j}\right) A_{1}^{-1}\right]+Y^{1},
$$

where

$$
Y^{j}=\sum_{s=(j-1) l+1}^{j l} E Y_{s}^{t}=E V_{t}^{j}, \quad j=1,2, \ldots, k .
$$

Consequently,

$$
\tilde{X}^{1}\left[d-I\left(\sum_{j=1}^{k} \tilde{X}^{j}\right) A_{1}^{-1}\right] \leq Y^{1} .
$$

Since $Y^{1}=A_{1}^{-1} E \theta E \gamma$ and $E \theta<\Delta c^{\prime}$, we conclude that $Y^{1} \rightarrow 0$ as $\Delta \rightarrow 0$. Also, by (12), the value $\tilde{X}^{1}$ is bounded from below and, by (10),

$$
\sum_{j=1}^{k} \tilde{X}^{j}-\tilde{X}^{1} \rightarrow 0 \quad \text { as } \quad \Delta \rightarrow 0 .
$$

Hence,

$$
I\left(\tilde{X}^{1}+o_{\Delta}(1)\right) A_{1}^{-1}-d \rightarrow 0 \quad \text { as } \quad \Delta \rightarrow 0,
$$

which, by continuity of $I(\cdot)$, implies (11). Here $o_{\Delta}(1) \rightarrow 0$ as $\Delta \rightarrow 0$.

The lemma is proved.

This lemma allows for the following conceptual conclusions. We see that if initial capitals vanish, then the most efficient firms dominate the industry. Their total productive capacity approaches $Q^{A_{1}}$, while the total productive capacity of all other firms vanishes.

Assume that $\tilde{\mathbf{x}}$ exists for some $\epsilon>0$. Since

$$
1-d+I\left(\sum_{i=1}^{\infty} \tilde{x}_{i}\right) A_{j}^{-1}<1, \quad j=1,2, \ldots, k,
$$

we conclude that for every $j$ and $p$ there is a number of cohort $s(j, p)+1$ such that

$$
\tilde{x}_{s(j, p) k l+l(j-1)+p}>0 \text { and } \tilde{x}_{s k l+l(j-1)+p}=0 \text { for } s>s(j, p) .
$$

We observe that, if the inflow of capitals vanishes in the sense specified above, then, for every $p$, the value $s(j, p)$ does not change much for $j=2,3, \ldots, k$, while $s(1, p)$ unboundedly increases. 
Indeed, by (10) and (11),

$$
\sum_{j=1}^{k} \tilde{X}^{j} \rightarrow Q^{A_{1}} \text { as } \Delta \rightarrow 0 .
$$

Thus, the factor driving the decrease of productive capacity in the number of cohorts does not change much for all but the most efficient firms. For the most efficient firms the factor approaches 1 as $\Delta \rightarrow 0$. Hence, if $q(t)$ concentrates as $t \rightarrow \infty$ close to some deterministic vector, then in this equilibrium the life time of the most efficient firms goes to infinity as capitals of new entering firms vanish, while life times of all other firms do not essentially change.

So far, we have showed that, if a solution $\tilde{\mathbf{x}}$ of (5) exists, it has certain properties. Now we shall look at the issue of existence of such solution.

Let $\epsilon=0$. By (7) we see that

$$
\tilde{X}^{j}=\tilde{X}^{j}\left[1-d+I\left(\sum_{i=1}^{k} \tilde{X}^{i}\right) A_{j}^{-1}\right]+Y^{j}, \quad j=1,2, \ldots, k,
$$

or

$$
\tilde{X}^{j}\left[d-I\left(\sum_{i=1}^{k} \tilde{X}^{i}\right) A_{j}^{-1}\right]=Y^{j}, \quad j=1,2, \ldots, k .
$$

Now, if we managed to prove that for every positive reals $y_{j}, j=1,2, \ldots, k$, the equations

$$
x_{j}\left[d-I\left(\sum_{i=1}^{k} x_{i}\right) A_{j}^{-1}\right]=y_{j}, \quad j=1,2, \ldots, k,
$$

have a single solution $\mathbf{x}^{*}$ with positive coordinates, this would imply that $\tilde{X}^{j}, \quad j=$ $1,2, \ldots, k$, always exist and are uniquely defined. By (6) and (7) this gives that the required $\tilde{\mathbf{x}}$ exists and is uniquely defined.

The following result, which is of the Fixed Point Theorem type, shows that there is indeed a single solution $\mathbf{x}^{*}$ of (13) with positive coordinates. (Notice that only such solution makes sense in the context of our model of industry evolution).

Lemma 3. Consider equations (13) for given reals $y_{j}>0, j=1,2, \ldots, k$. There is a single solution $\mathbf{x}^{*}$ of it with $x_{j}^{*}>0, j=1,2, \ldots, k$.

Proof. For $v>0$ consider the equation

$$
v=T(v)
$$

where

$$
T(v)=\sum_{j=1}^{k} \frac{y_{j}}{d-I(v) A_{j}^{-1}} .
$$


Notice that

$$
\begin{aligned}
& v-T(v)>0 \text { for small } v>0, \quad v-T(v) \rightarrow \infty \text { as } v \rightarrow \infty, \\
& v-T(v) \rightarrow-\infty \text { as } v \rightarrow Q^{A_{j}}-, \quad v-T(v) \rightarrow \infty \text { as } v \rightarrow Q^{A_{j}}+,
\end{aligned}
$$

where $j=1,2, \ldots, k$. By continuity of functions involved, these relations allow to conclude that on $\left(0, Q^{A_{k}}\right),\left(Q^{A_{i+1}}, Q^{A_{i}}\right), i=1,2, \ldots, k-1$, and $\left(Q^{A_{1}}, \infty\right)$ there are roots $\eta^{i}$ (possibly not a single one for some of the intervals) of equation (14).

If $\mathbf{x}^{\prime}$ is a solution of (13), then

$$
x_{j}^{\prime}=\frac{y_{j}}{d-I\left(\sum_{i=1}^{k} x_{i}^{\prime}\right) A_{j}^{-1}}, j=1,2, \ldots, k .
$$

and

$$
\sum_{i=1}^{k} x_{i}^{\prime}
$$

is a solution of (14). Let

$$
\sum_{j=1}^{k} x_{j}^{\prime} \in\left(0, Q^{A_{k}}\right),
$$

then by (15) we see that $x_{j}^{\prime}<0$ for $j=1,2, \ldots, k$. Hence, this root having all negative coordinates, cannot be $\mathbf{x}^{*}$. Assume now that

$$
\sum_{j=1}^{k} x_{j}^{\prime} \in\left(Q^{A_{i+1}}, Q^{A_{i}}\right)
$$

for some $i=1,2, \ldots, k-1$. By (15) we get $x_{j}^{\prime}<0$ for $j=1,2, \ldots, i$. Consequently, this root has some of the coordinates negative. Hence, it cannot be $\mathbf{x}^{*}$ either. Now let

$$
\sum_{i=1}^{k} x_{i}^{\prime} \in\left(Q^{A_{1}}, \infty\right) .
$$

Then, by (15), $x_{j}^{\prime}>0, j=1,2, \ldots, k$. Thus, we have showed that if there is a solution of (13) with positive coordinates, the sum of coordinates must belong to $\left(Q^{A_{1}}, \infty\right)$. Let us show that this solution exists.

If $v>Q^{A_{1}}$, the left hand side of (14) increases in $v$, while the right hand side is a decreasing function. Hence there is a single solution $\eta^{1}$ of $(14)$ on $\left(Q^{A_{1}}, \infty\right)$. Set

$$
x_{j}\left(\eta^{1}\right)=\frac{y_{j}}{d-I\left(\eta^{1}\right) A_{j}^{-1}}, j=1,2, \ldots, k .
$$


Then $\mathbf{x}\left(\eta^{1}\right)$ has all positive coordinates and satisfies (13). Thus, a required solution exists. It is unique. Indeed, let $\mathbf{x}^{\prime}$ be any other solution with positive coordinates. Since we have showed that (14) has a single solution on $\left(Q^{A_{1}}, \infty\right)$, we conclude that

$$
\sum_{j=1}^{k} x_{j}^{\prime}=\eta^{1}
$$

Hence, by (16) $\mathbf{x}^{\prime}$ must coincide with $\mathbf{x}\left(\eta^{1}\right)$.

The lemma is proved.

We conclude that if there is no mortality, equation (5) always has a single solution $\tilde{\mathbf{x}}$. This is the only candidate for the long run deterministic equilibrium of process (3). The following argument shows that this is not, in general, the case if $\epsilon>0$. Namely, one cannot prove that there is always a solution of (5). Hence, there is no natural candidate for a long run deterministic equilibrium. In fact, such equilibrium should not be expected, in general.

Indeed, let $\epsilon>0$. Setting

$$
\tilde{X}^{j, p}=\sum_{i=0}^{s(j, p)} \tilde{x}_{i k l+l(j-1)+p}, \quad j=1,2, \ldots, k, \quad p=1,2, \ldots, l,
$$

we see, by (6) and (7), that

$$
\tilde{X}^{j, p}=\tilde{X}^{j, p}\left[1-d+I\left(\sum_{i=1}^{k} \sum_{q=1}^{l} \tilde{X}^{i, q}\right) A_{j}^{-1}\right]+Y^{j, p}\left\{1-\left[1-d+I\left(\sum_{i=1}^{k} \sum_{q=1}^{l} \tilde{X}^{i, q}\right) A_{j}^{-1}\right]^{s(j, p)}\right\},
$$

where positive integers $s(j, p)$ are determined as above and, also, $Y^{j, p}=E Y_{(j-1) l+p}^{t}$ for all possible $j$ and $p$. Now, to prove the existence of $\tilde{\mathbf{x}}$, we have to consider the following equations with respect to $x^{j, p}$

$$
x^{j, p}\left[d-I\left(\sum_{i=1}^{k} \sum_{q=1}^{l} x^{i, q}\right) A_{j}^{-1}\right]=Y^{j, p}\left\{1-\left[1-d+I\left(\sum_{i=1}^{k} \sum_{q=1}^{l} x^{i, q}\right) A_{j}^{-1}\right]^{s(j, p)}\right\},
$$

where $j=1,2, \ldots, k, p=1,2, \ldots, l$ and $s(j, p)$ designates a positive integer that depends on

$$
\sum_{i=1}^{k} \sum_{q=1}^{l} x^{i, q}
$$

in such a way that $s(j, p)=\infty$ if

$$
\left.1-d+I\left(\sum_{i=1}^{k} \sum_{q=1}^{l} x^{i, q}\right) A_{j}^{-1}\right] \geq 1
$$

and

$$
s(j, p)=\max s: \quad Y^{j, p} A_{j}\left[1-d+I\left(\sum_{i=1}^{k} \sum_{q=1}^{l} x^{i, q}\right) A_{j}^{-1}\right]^{s} \geq \epsilon b
$$


if

$$
\left.1-d+I\left(\sum_{i=1}^{k} \sum_{q=1}^{l} x^{i, q}\right) A_{j}^{-1}\right]<1 .
$$

Since the expressions involved in the right hand sides of (17) are discontinuous functions of

$$
\sum_{i=1}^{k} \sum_{q=1}^{l} x^{i, q}
$$

we cannot use here the argument employed in Lemma 3 to show that equations (17) have a solution.

To summarize: in a nutshell, while a candidate for a deterministic limit can be shown to exist - under the assumption of no mortality - that corresponds to the (rather peculiar) circumstances where inflows of productive capacity through entry exactly matches the (negative) net investment by incumbents ${ }^{5}$. Moreover, all firms have "atomic" sizes. While little descriptive value should be attributed to the existence result - given the restrictions on which it is based -, it can be used to assess some informal properties of "comparative dynamics", which, to repeat, hold rigorously only when $\mathbf{q}(t)$ approaches a deterministic limit (inevitably $\tilde{\mathbf{x}}$ ) as $t \rightarrow \infty$. Otherwise, take what immediately follows as an exercise of "what ... if" character, or, with an undefined degree of arbitrariness, as revealing some qualitative features of the dynamics also under less restrictive conditions.

With these caveats in mind let us consider some properties of the limit productive capacity $Q^{*}$ on the parameters of the model in the case when $\epsilon=0$.

Set

$$
Q^{*}=\sum_{j=1}^{k} \tilde{X}^{j}=E \theta E \gamma \sum_{j=1}^{k}\left[d A_{j}-I\left(Q^{*}\right)\right]^{-1},
$$

then

$$
\tilde{X}^{j}=\frac{E \theta E \gamma}{d A_{j}-I\left(Q^{*}\right)}, j=1,2, \ldots, k
$$

The expression given above for $Q^{*}$ defines it as an implicit function of the parameters involved in the model, namely, $\lambda, v, m, d, E \theta, E \gamma, A_{i}, i=1,2, \ldots, k$. Let us calculate the partial derivatives of $Q^{*}$ on them to assess on what is the influence of these variables on

\footnotetext{
${ }^{5}$ Note that, not too surprisingly, the above results turn out to be somewhat comparable with those obtained in a mode of analysis whereby one imputes to agents an ex ante rationality sophisticated enough as to sustain, self-fulfillingly, equilibrium paths themselves (cf., for example, Jovanovic (1982) and Hopenheyn (1992)).
} 
$Q^{*}$. Hence, the analysis is restricted to the domain where $I(\cdot)$ is a differentiable function of $Q^{*}$. Note, that $I(x) \equiv 0$ is a differentiable function for $x>\bar{Q}$. (Recall that $\bar{Q}=h^{-1}(m)$ ). Hence, for $Q^{*}>\bar{Q}$ the following argument always applies. (If $Q^{*}<\bar{Q}$, we have to require differentiability of the demand function.) By relations (10) and (11), $Q^{*}<\bar{Q}$ if, for example, $E \theta$ is small enough. Indeed, the latter implies that $Q^{*}$ is close to $Q^{A_{1}}$, but $Q^{A_{1}}<\bar{Q}$.

Remark 1. Note that if $Q^{*}=\bar{Q}$ we get

$$
m=E \theta E \gamma \sum_{j=1}^{k} A_{j}^{-1} .
$$

Indeed, a situation such as when $Q^{*}=\bar{Q}$ appears to be rather inlikely: given all other

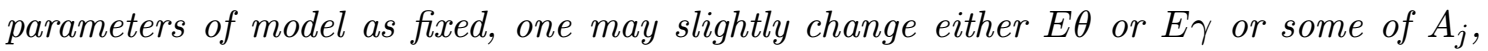
$j=1,2, \ldots$, in such a way that this equality never holds. Conversely, specifying the parameters of the model, we can require that it does not take place.

We get

$$
\frac{\partial Q^{*}}{\partial \alpha}\left\{1-\alpha \beta I^{\prime}\left(Q^{*}\right) \sum_{i=1}^{k}\left[d A_{i}-I\left(Q^{*}\right)\right]^{-2}\right\}=\beta \sum_{i=1}^{k}\left[d A_{i}-I\left(Q^{*}\right)\right]^{-1},
$$

where $I^{\prime}(x)=\partial I(x) / \partial x=\lambda h^{\prime}(x) / v r$ if $x<\bar{Q}$ and $I^{\prime}(x)=0$ if $x>\bar{Q}$ (also remember that $\alpha=E \theta$ and $\beta=E \gamma)$. The demand function decreases, hence, $h^{\prime}(\cdot)<0$. Thus, we conclude that $\frac{\partial Q^{*}}{\partial \alpha}>0$. Similarly, $\frac{\partial Q^{*}}{\partial \beta}>0$. Also

$$
\frac{\partial Q^{*}}{\partial A_{j}}\left\{1-\alpha \beta d I^{\prime}\left(Q^{*}\right) \sum_{i=1}^{k}\left[d A_{i}-I\left(Q^{*}\right)\right]^{-2}\right\}=-\alpha \beta\left[A_{j}-I\left(Q^{*}\right)\right]^{-2},
$$

which implies that

$$
\frac{\partial Q^{*}}{\partial A_{j}}<0, j=1,2, \ldots, k \text {. }
$$

Similarly, $\frac{\partial Q^{*}}{\partial d}<0$. Furthermore, if $Q^{*}<\bar{Q}$, then $\frac{\partial Q^{*}}{\partial \lambda}>0, \frac{\partial Q^{*}}{\partial v}<0$ and $\frac{\partial Q^{*}}{\partial m}<0$ while $\frac{\partial Q^{*}}{\partial \lambda}=0, \frac{\partial Q^{*}}{\partial v}=0$ and $\frac{\partial Q^{*}}{\partial m}=0$ while for $Q^{*}>\bar{Q}$.

Conceptually, the obtained inequalities can be interpreted in the following way. The "steady-state" (that is, in the limit, the "equilibrium" capacity) total productive capacity is positively related to the value of the average capital of new entrants and to the average number of them. (Intuitively, the result supports the idea, generally put forward in more static setting that easier conditions of entry yield lower prices and lower profits - other things being equal and with monotonically falling demands). It is somewhat less intuitive, however, that productive capacity is negatively related to each of the values of capital per 
unit of output and to depreciation rate - given that one does not allow for any "barriers to entry" or indivisibility in the investment costs. Moreover, productive capacity is related positively with the propensity to invest and negatively with the value of price per unit of physical capital and to the value of variable costs per unit of output, provided that the equilibrium price $h\left(Q^{*}\right)$ exceeds the variable costs per unit of output. Otherwise, if the equilibrium price equals to the variable costs per unit of output, the total steady-state productive capacity does not depend on these three variables.

(As already noted, those observations can be symmetrically reformulated in terms of the equilibrium price $\left.h\left(Q^{*}\right)\right)$.

Remark 2. The share in the total equilibrium productive capacity of all firms having $A_{j}$ as capital per unit of output reads

$$
s_{j}^{*}=\frac{\tilde{X}^{j}}{Q^{*}}=\frac{1}{1+\sum_{i=1, i \neq j}^{k}\left[A_{i}-I\left(Q^{*}\right)\right]^{-1}} .
$$

Also, the ratio of the total equilibrium productive capacity of all firms having $A_{i}$ as capital per unit of output to the one of all firms firms with $A_{j}$ as capital per unit of output is

$$
\frac{s_{i}^{*}}{s_{j}^{*}}=\frac{A_{j}-I\left(Q^{*}\right)}{A_{i}-I\left(Q^{*}\right)} .
$$

Notice that this ratio is $A_{j} / A_{i}$ as soon as $Q^{*} \geq \bar{Q}$. As above, we can treat $s_{j}^{*}$ as an implicit function of parameters involved in the model. Thus, taking partial derivatives, we can infer the influence of them on $s_{j}^{*}$.

\section{$5 \quad$ Entry, Mortality and Long Run Balance Relations}

As the foregoing analysis shows, there is little hope of proving convergence of the vectors $\mathbf{q}(t)$ to a limit as $t \rightarrow \infty$. However, one way try to study another (weaker) type of convergence, namely, convergence with probability one of time averages of these vectors.

Start from the statement that in a finite time with probability one there will be born at least one firm with $A_{j}$ as capital per unit of output.

Lemma 4. For each $j=1,2, \ldots, k$ in a finite random time $\tau_{j}$ with probability one there appears a firm with $A_{j}$ as capital per unit of output.

Proof. Note that, since the variable $\gamma$ is not deterministic, then $P\{\gamma=0\}=p_{0}<1$. Also,

$$
\left\{\tau_{j}=\infty\right\}=\bigcap_{n \geq 0}\left\{\tau_{j}>n\right\}
$$


Hence

$$
P\left\{\tau_{j}=\infty\right\}=P\left\{\bigcap_{n \geq 0}\left\{\tau_{j}>n\right\}\right\} .
$$

Since $\left\{\tau_{j}>n\right\} \supseteq\left\{\tau_{j}>n+1\right\}$, we have

$$
P\left\{\bigcap_{n \geq 0}\left\{\tau_{j}>n\right\}\right\}=\lim _{n \rightarrow \infty} P\left\{\tau_{j}>n\right\} .
$$

But

$$
P\left\{\tau_{j}>n\right\}=p_{0}^{k n} \rightarrow 0 \quad \text { as } n \rightarrow \infty .
$$

This completes the proof.

Now let us show that $Q_{t}^{A_{j}}, j=1,2, \ldots, k$, are bounded with certainty. Since $I(x)$ decreases to 0 as $x$ increases, there is $Q_{*}^{j} \geq Q^{A_{j}}$ such that

$$
\left[d-I(x) A_{j}^{-1}\right] x \geq l c A_{j}^{-1} \text { for } x \geq Q_{*}^{j} .
$$

If $Q_{t}^{A_{j}}$ were to grow to infinity, then for some finite time instant $t_{0}>1$

$$
Q_{t_{0}}^{A_{j}} \geq Q_{*}^{j}
$$

Dropping the nonpositive term in (1), we get

$$
Q_{t_{0}+1}^{A_{j}} \leq Q_{t_{0}}^{A_{j}}\left[1-d+I\left(Q_{t_{0}}\right) A_{j}^{-1}\right]+V_{t_{0}}^{j} .
$$

Since $I(\cdot)$ is a decreasing function, then $I\left(Q_{t_{0}}\right) \leq I\left(Q_{t_{0}}^{A_{j}}\right)$. Also notice that $V_{t_{0}}^{j} \leq l c / A_{j}$. Hence, the above inequality and (18) imply

$$
Q_{t_{0}+1}^{A_{j}} \leq Q_{t_{0}}+Q_{t_{0}}^{A_{j}}\left[I\left(Q_{t_{0}}^{A_{j}}\right) A_{j}^{-1}-d\right]+l c A_{j}^{-1} \leq Q_{t_{0}}^{A_{j}}
$$

Thus, $Q_{t}^{A_{j}}$ cannot grow to infinity. This value is bounded with certainty from above and the bound does not exceed $Q_{*}^{j}$.

In the same way, setting

$$
\bar{Q}=h^{-1}(m), \quad \hat{Q}=\min x \geq \bar{Q}: \quad d x \geq l c \sum_{j=1}^{k} A_{j}^{-1}
$$

and

$$
Q_{*}=\min \left(\hat{Q}, \sum_{j=1}^{k} Q_{*}^{j}\right),
$$

we show that $Q_{t}$ is bounded with certainty by a constant which does not exceed $Q_{*}$.

Let us formulate these results in a separate statement. 
Lemma 5. With certainty $Q_{t}^{A_{j}} \leq Q_{*}^{j}, j=1,2, \ldots, k$, and $Q_{t} \leq Q_{*}$, where the constants are given by (18) and (19).

The next statement gives an alternative estimate for the total productive capacity.

Lemma 6. With probability one $\check{Q} \geq Q^{A_{1}}$, where $\check{Q}=\lim \sup _{t \rightarrow \infty} Q_{t}$.

Proof. By Lemma 5 the sequence $Q_{t}, t \geq 1$, is bounded with certainty, consequently the random variable $\check{Q}$ exists. Assume to the contrary that $\check{Q}<Q^{A_{1}}$ with positive probability. Then there is $\delta>0$ such that $P\left\{\Omega_{\delta}\right\}>0$, where $\Omega_{\delta}=\left\{\omega \in \Omega: \check{Q}<Q^{A_{1}}-\delta\right\}$. For every elementary outcome $\omega \in \Omega_{\delta}$ there is $t(\omega)$ such that $Q_{t} \leq Q^{A_{1}}-\delta$ for $t \geq t(\omega)$. Then for $t \geq \max \left[t(\omega), \tau_{1}(\omega)\right]$ (that is, we want to be sure here that firms with $A_{1}$ as capital per unit of outputt are present in the industry) we get by (1)

$$
Q_{t+1}^{A_{1}} \geq(1+\rho) Q_{t}^{A_{1}}
$$

where $\rho=I\left(Q^{A_{1}}-\delta\right) A_{1}^{-1}-d>0$. We took into account here that $Q_{t}<Q^{A_{1}}-\delta<Q^{A_{1}}$, which implies that all firms with $A_{1}$ as capital per unit of output continue to grow for $t \geq \max \left[t(\omega), \tau_{1}(\omega)\right]$. Inequality (20) implies unbounded growth of $Q_{t}^{A_{1}}$ as $t \rightarrow \infty$. Thus, $Q_{t}^{A_{1}}, t \geq 1$, are not bounded on $\Omega_{\delta}$. On the other hand, by Lemma 5 this sequence is bounded with certainty. This is a contradiction. Thus, the assumption that $P\left\{\Omega_{\delta}\right\}>0$ contradicts the boundness (with certainty) of $Q_{t}^{A_{1}}, t \geq 1$. Hence, $P\left\{\Omega_{\delta}\right\}=0$ for every $\delta>0$, which implies that $P\left\{\check{Q} \geq Q^{A_{1}}\right\}=1$.

The lemma is proved.

Let us now show that, if there is a death threshold, then none of the firms can survive for infinitely long time.

Theorem 1. If $\epsilon>0$ and $P\{\gamma=0\}=p_{0}=0$, then each firm dies in a finite random time with probability one.

Proof. The death threshold implies that if a firm lives infinitely long, then its capital does not drop below $\epsilon b$. Since the total productive capacity of the industry is bounded with certainty, we conclude that starting from a finite random time $\tau$ with probability one every newcoming firm dies in a finite time. Indeed, otherwise we would have infinitely many firms living infinitely long. This, by boundness from below of their capitals, would imply that the total productive capacity goes to infinity.

At time $t \geq \tau$ consider two firms: one with capital $c_{t}$ and capital per unit of output $A_{i}$ and the other with capital $c_{t}^{\prime}$ and capital per unit of output $A_{j} \leq A_{i}$. Then

$$
\frac{c_{t+1}}{c_{t+1}^{\prime}}=\frac{c_{t}\left[1-d+I\left(Q_{t}\right) A_{i}^{-1}\right] \chi_{c_{t}\left[1-d+I\left(Q_{t}\right) A_{i}^{-1}\right] \geq \epsilon b}}{c_{t}^{\prime}\left[1-d+I\left(Q_{t}\right) A_{j}^{-1}\right] \chi_{c_{t}^{\prime}\left[1-d+I\left(Q_{t}\right) A_{j}^{-1}\right] \geq \epsilon b}} .
$$


Assume that there is a firm living infinitely long with positive probability. Set $c_{t}$ for its capital at $t$ and $A_{i}$ for its capital per unit of output. Then

$$
P\left\{c_{t} \geq \epsilon b, t \geq \tau^{\prime}\right\}=\delta>0,
$$

where $\tau^{\prime}$ stands for the time instant when it comes to the industry. Consider a time instant $t \geq \tau$. There is a most efficient firm coming at $t$. Set $c_{t}^{\prime}$ for its capital. Since we are in the time domain where every entrant dies in a finite time, also this most efficient firm dies at a finite time instant $t^{\prime}>t$ with probability one. By (21) we get that

$$
c_{t^{\prime}} \leq c_{t} \frac{c_{t^{\prime}}^{\prime}}{c_{t}} \leq c_{t} \frac{\epsilon b}{b} \leq \epsilon c_{t} .
$$

Since $p_{0}=0$, at $t^{\prime}$ another most efficient firm comes to the industry. Similarly, it dies at some instant $t^{\prime \prime}$ and we obtain that $c_{t^{\prime \prime}} \leq \epsilon c_{t^{\prime}}$ or $c_{t^{\prime \prime}} \leq \epsilon^{2} c_{t}$.

If $\epsilon<1$, we conclude that, since $c_{t}$ is uniformly bounded from above (cf. Lemma 5), there is a sequence $t_{k}, k \geq 1$, of random time instants such that with certainty $c_{t_{k}} \rightarrow 0$ as $k \rightarrow \infty$. This contradicts (22). Hence, it is not possible that there is a firm surviving infinitely long with positive probability.

If $\epsilon=1$, we notice that, since $\theta$ is not deterministic, there is $\sigma>0$ such that $P\{\theta \geq$ $b+\sigma\}>0$. By an argument similar to the one given in the proof of Lemma 4 , we conclude that with probability one there is a sequence of random time instants $t_{k}^{\prime}, k \geq 1$, such that at least one of the most efficient firms born at $t_{k}^{\prime}$ has initial capital exceeding $b+\sigma$. Then for $t_{k} \geq \tau$ we have that the capital of the infinitely long living firm at least does not grow (for the previous argument), but it shrinks at least $1+\sigma / b$ times during the life time of every new most efficient entrant whose initial capital is equal or greater than $b+\sigma$. Since with probability one there are infinitely many of the latter firms, this again contradict the assumption that such firm can live forever.

The theorem is proved.

From (1) we get that

$$
Q_{t+1}^{A_{j}}-Q_{t}^{A_{j}}=Q_{t}^{A_{j}}\left[I\left(Q_{t}\right) A_{j}^{-1}-d\right]+V_{t}^{j}-\mathcal{E}_{t}^{j} .
$$

This implies for $n \geq 1$

$$
Q_{n+1}^{A_{j}}-Q_{1}^{A_{j}}=\sum_{t=1}^{n}\left\{Q_{t}^{A_{j}}\left[I\left(Q_{t}\right) A_{j}^{-1}-d\right]+V_{t}^{j}-\mathcal{E}_{t}^{j}\right\} .
$$

Since $Q_{t}^{A_{j}}, t \geq 1$, are bounded with certainty, then by (23) we conclude that (with certainty)

$$
\frac{1}{n} \sum_{t=1}^{n}\left\{Q_{t}^{A_{j}}\left[I\left(Q_{t}\right) A_{j}^{-1}-d\right]+V_{t}^{j}-\mathcal{E}_{t}^{j}\right\} \rightarrow 0
$$


as $n \rightarrow \infty$. Due to the strong law of large numbers, with probability one

$$
\frac{1}{n} \sum_{t=1}^{n} V_{t}^{j} \rightarrow A_{j}^{-1} E \gamma E \theta
$$

as $n \rightarrow \infty$. Here by $E \gamma$ and $E \theta$ we designate the mean values of $\gamma$ and $\theta$. By (24) and (25) we conclude the following.

Lemma 7. For $j=1,2, \ldots, k$ with probability one

$$
\frac{1}{n} \sum_{t=1}^{n}\left\{Q_{t}^{A_{j}}\left[d-I\left(Q_{t}\right) A_{j}^{-1}\right]+\mathcal{E}_{t}^{j}\right\} \rightarrow a A_{j}^{-1}
$$

as $n \rightarrow \infty$. Here $a=E \gamma E \theta, j=1,2, \ldots, k$.

Summing up relations (26), we get that with probability one

$$
\frac{1}{n} \sum_{t=1}^{n}\left[d Q_{t}-I\left(Q_{t}\right) \sum_{j=1}^{k} Q_{t}^{A_{j}} A_{j}^{-1}+\mathcal{E}_{t}\right] \rightarrow a \sum_{j=1}^{k} A_{j}^{-1} .
$$

as $n \rightarrow \infty$. Relations (26) and (27) represent the most general long run balance equation for the productive capacities involved in the market. They imply that

$$
Q_{t}^{A_{j}}\left[d-I\left(Q_{t}\right) A_{j}^{-1}\right]+\mathcal{E}_{t}^{j}-a A_{j}^{-1}, j=1,2, \ldots, k,
$$

and

$$
d Q_{t}-I\left(Q_{t}\right) \sum_{j=1}^{k} Q_{t}^{A_{j}} A_{j}^{-1}+\mathcal{E}_{t}^{j}-a \sum_{j=1}^{k} A_{j}^{-1}
$$

fluctuate through time in such a way that on average positive deviations of these values from zero are compensated by their negative deviations.

However, the results given by Lemma 7 do not say anything about the limit behavior of time averages of $Q_{t}^{A_{j}}$ or $Q_{t}$. To study this issue, let us turn to the ergodic properties of process (3).

\section{Ergodic Properties of the Industry}

Define $\mathcal{B}^{\infty}$ the minimal $\sigma$-field in $R^{\infty}$ generated by sets of the following form

$$
A=\bigotimes_{j=1}^{\infty} A^{j}
$$

where $A^{j}$ designates a set from the $\sigma$-field of Borel sets $\mathcal{B}_{j}^{k l}$ in $R_{j}^{k l}$. For every such set $A$ one step transition probability of process $(3)$ reads

$$
p^{1}(\mathbf{q}, A)=P\{\mathbf{D}(\mathbf{q})+\mathbf{Y} \in A\}=P\left\{\mathbf{Y}^{*} \in A^{1}\right\} \chi_{\mathbf{D}(\mathbf{q}) \in \bigotimes_{i=2}^{\infty} A^{i}}
$$


Here $\mathbf{Y}^{*}$ stands for the $k l$-dimensional vector whose coordinates coincide with first $k l$ coordinates of a generic vector $\mathbf{Y}$ having the same distribution as $\mathbf{Y}^{t}, t \geq 0$. The total productive capacity is bounded with certainty. Consequently, process (3) belongs with probability one to

$$
L=\left\{\mathbf{q} \in R^{\infty}: \sum_{i=1}^{\infty} q_{i} \leq 2 Q_{*}, q_{i} \geq 0, i \geq 1\right\}
$$

To study the ergodic properties of process (3), we need the following condition, due to Doeblin (see Doob (1953), p. 192): there is a finite positive measure $\phi(\cdot)$ with $\phi(L)>0$ and a number $\epsilon \in(0,1)$ such that for all $\mathbf{q} \in L$

$$
p^{1}(\mathbf{q}, A) \leq 1-\epsilon \text { if } \phi(A) \leq \epsilon
$$

For a set $A$ as in (28) let $\phi(A)=P\left\{Y^{*} \in A^{1}\right\}$. Then, by (29), we see that $p^{1}(\mathbf{q}, A) \leq$ $\phi(A)$. Hence, restricting ourselves to $\epsilon<1 / 2$, we see that if $\phi(A) \leq \epsilon$, then $p^{1}(\mathbf{q}, A) \leq$ $\epsilon \leq 1-\epsilon$. Thus, Doeblin's condition holds for this choice of $\phi(\cdot)$ and all $\epsilon<1 / 2$.

Now, by Theorem 5.7 (Doob (1953), p. 214), we see that

$$
\pi(\mathbf{q}, A)=\lim _{n \rightarrow \infty} \frac{1}{n} \sum_{t=1}^{n} p^{t}(\mathbf{q}, A)
$$

defines for each $\mathbf{q} \in L$ a stationary absolute distribution. Here $p^{t}(\mathbf{x}, \cdot)$ stands for the transition probability in $t$ steps, that is,

$$
p^{t}(\mathbf{q}, A)=\int_{L} p^{t-1}(\mathbf{y}, A) d p^{1}(\mathbf{q}, \mathbf{y}), t \geq 2 .
$$

The stationary distribution $\pi(\mathbf{q}, \cdot)$ turns out to be the same, that is $\pi_{£}(\cdot)$, for all $\mathbf{q}$ belonging to the same ergodic set $£$ (see Doob (1953), p. 210). It has the following generic property

$$
\int_{£} p^{1}(\mathbf{x}, A) d \pi_{£}(\mathbf{x})=\pi_{£}(A)
$$

In general, it is not possible to find from this relation an explicit expression for $\pi_{£}(\cdot)$.

Let $\rho(\cdot)$ be a function measurable with respect to $\mathcal{B}^{\infty}$ and integrable with respect to $\pi(\mathbf{q}, \cdot)$. By the strong law of large numbers (see Doob (1953), p. 220), as $n \rightarrow \infty$

$$
\frac{1}{n} \sum_{t=0}^{n} \rho(\mathbf{q}(t)) \rightarrow \int_{L} \rho(\mathbf{x}) d \pi(\mathbf{q}, \mathbf{x})
$$

with probability one, provided that the process (3) starts at a deterministic point q. This result shows that all sensible time averages of process (3) converge with probability one to deterministic limits (given a deterministic initial state). In our particular case $\mathbf{q}(0)$ is 
always $\mathbf{Y}^{0}$. Hence, the time averages our process are deterministic functions of $\mathbf{Y}^{0}$ (or, equivalently, of $\left.\mathbf{Y}^{*}\right)$.

Thus, the following statement holds.

Theorem 2. With probability one

$$
\begin{aligned}
& \frac{1}{n} \sum_{t=1}^{n} Q_{t}^{A_{j}} \rightarrow \int_{L} \sum_{i=1}^{\infty} \sum_{p=1}^{l} x_{i k l+(j-1) l+p} d \pi\left(\mathbf{Y}^{0}, \mathbf{x}\right), \\
& \frac{1}{n} \sum_{t=1}^{n} Q_{t}^{A_{j}} I\left(Q_{t}\right) \rightarrow \int_{L} I\left(\sum_{i=1}^{\infty} x_{i}\right) \sum_{s=1}^{\infty} \sum_{p=1}^{l} x_{s k l+(j-1) l+p} d \pi\left(\mathbf{Y}^{0}, \mathbf{x}\right), \\
& \frac{1}{n} \sum_{t=1}^{n} \nu^{j}(t) \rightarrow \int_{L} \sum_{i=1}^{\infty} \sum_{p=1}^{l} \chi_{x_{i k l+(j-1) l+p} A_{j} \geq \epsilon b} d \pi\left(\mathbf{Y}^{0}, \mathbf{x}\right)
\end{aligned}
$$

as $n \rightarrow \infty$. Here $j=1,2, \ldots, k$. By $\nu^{j}(t)$ we designate the number of firms having $A_{j}$ as capital per unit of output which are manufacturing at time $t$. Also, $\pi\left(\mathbf{Y}^{0}, \cdot\right)$ designates a random measure taking the value $\pi_{£}(\cdot)$ for an elementary outcome $\omega \in \Omega$ as soon as $\mathbf{Y}^{0}$ belongs for this $\omega$ to an ergodic set $£$.

Proof. The sums involved in (30) - (32) are measurable with respect to $\mathcal{B}^{\infty}$ nonnegative functions. Indeed, for (30) and (32) it is obvious, for (31) we have to take into account that $I(\cdot)$ is a continuous function by hypothesis. By definition of $L$, the sum involved in (30) is uniformly bounded from above by $2 Q_{*}$. Taking into account the continuity of $I(\cdot)$, we get that the expression in the right hand side of (31) is bounded from above by

$$
2 Q_{*} \max _{x \in\left[0,2 Q_{a} s t\right]} I(x)<\infty .
$$

Notice that the minimal productive capacity of a firm with $A_{j}$ as capital per unit of output which is manufacturing is $\epsilon b A_{j}^{-1}$. Since the total productive capacity of such firms does not exceed $Q_{*}^{j}$ for any time instant, we conclude that the iterated sum in (32) is bounded from above by $A_{j} Q_{*}^{j} / \epsilon$. Thus, the functions involved in the right hand sides of (30) - (32) are measurable and uniformly bounded. Consequently, they are integrable with respect to $\pi\left(\mathbf{Y}^{0}, \cdot\right)$, a random probability measure. It takes the value $\pi(\mathbf{y}, \cdot)$ if $\mathbf{Y}^{0}=\mathbf{y}$. Applying the strong law of large numbers quoted above, we obtain the statement of the theorem.

The theorem is proved.

Relations (30) - (32) allow for the following conceptual interpretations. The first one means that the time average of the total productive capacity of firms that are in business and have $A_{j}$ as capital per unit of output converges to a limit which is a deterministic function of $\mathbf{Y}^{0}$. Since

$$
\max \left[h\left(Q_{t}\right)-m, 0\right] Q_{t}^{A_{j}}=\frac{v}{\lambda} I\left(Q_{t}\right) Q_{t}^{A_{j}}
$$


represents the gross total profit of firms having $A_{j}$ as capital per unit of output at time $t$, the second relation says that the time average of this value converges to a limit being a deterministic function of $\mathbf{Y}^{0}$. The third relation means that the average number of firms with $A_{j}$ as capital per unit of output that are in business converges to a limit which is also a deterministic function of $\mathbf{Y}^{0}$.

From (26), (30) and (31) we get the following result.

Corollary. With probability one

$$
\frac{1}{n} \sum_{t=0}^{n} \mathcal{E}_{t}^{j}, \quad j=1,2, \ldots, k
$$

converge as $n \rightarrow \infty$ and the limits are deterministic functions of $\mathbf{Y}^{0}$. These limits satisfy the following equations

$$
e^{j}\left(\mathbf{Y}^{0}\right)=a A_{j}^{-1}-d q^{j}\left(\mathbf{Y}^{0}\right)+\frac{\lambda}{v} r^{j}\left(\mathbf{Y}^{0}\right) A_{j}^{-1}, \quad j=1,2, \ldots, k,
$$

where $q^{j}\left(\mathbf{Y}^{0}\right)$ and $r^{j}\left(\mathbf{Y}^{0}\right)$ designate the values in the right hand sides of (30) and (31).

Thus, this corollary gives expressions for the limit average total outflows of productive capacities of firms dependent on the other ergodic characteristics of the industry and the parameters of the model.

An important issue concerns the possible path-dependency of the dynamic. Doeblin condition implies that events occuring at $t$ and $t+n$ are getting more and more independent as $n \rightarrow \infty$. This ergodic property means as such lack of path-dependency. On the other hand the limits for the time averages for productive capacities, outflows of the latters, numbers of firms in business, etc. do depend on the initial state. But the dependency acts in such a way that the limits turn out to be the same for all initial states belonging to the same ergodic set. Hence, one can say that there is a "weak" form of path-dependency governed by a partition of $\Omega$ by ergodic sets. This partition, in general, turns out to be less fine than the one generated by $\mathbf{Y}^{0}$. The conditions of birth of the industry do not affect the limits of time averages only in those circumstances one is able to prove that there is a single ergodic set (and, hence, the limits do not depend on $\mathbf{Y}^{0}$ ).

Now let turn to numerical simulations of the model.

\section{Some Results of Computer Simulations}

As an illustration of some qualitative properties of the model, let us consider a computer 
simulation $^{6}$. The run presented below used the following parametrization: $k=2, A_{1}=1$, $A_{2}=3, m=1, v=1, d=0.3, \lambda=0.6, \epsilon=0.5$. The demand function was $h(x)=$ $4.1667 \exp (-0.5 x)$ and the capitals of newcoming firms were uniformly distributed over $[0.02,0.04]$. We set $l=3$ and the number of newcoming firms is characterized by the following distribution: $p_{0}=0.1, p_{1}=0.4, p_{2}=0.3$ and $p_{3}=0.2$.

Figure 1 shows the dynamic of the price for $1 \leq t \leq 50$ : it tends stabilize rather quickly around its long term average level, which indeed is below that value at which everybody shrinks. (Note that this is a property of the price average, while the actual price keeps fluctuating around this level.)

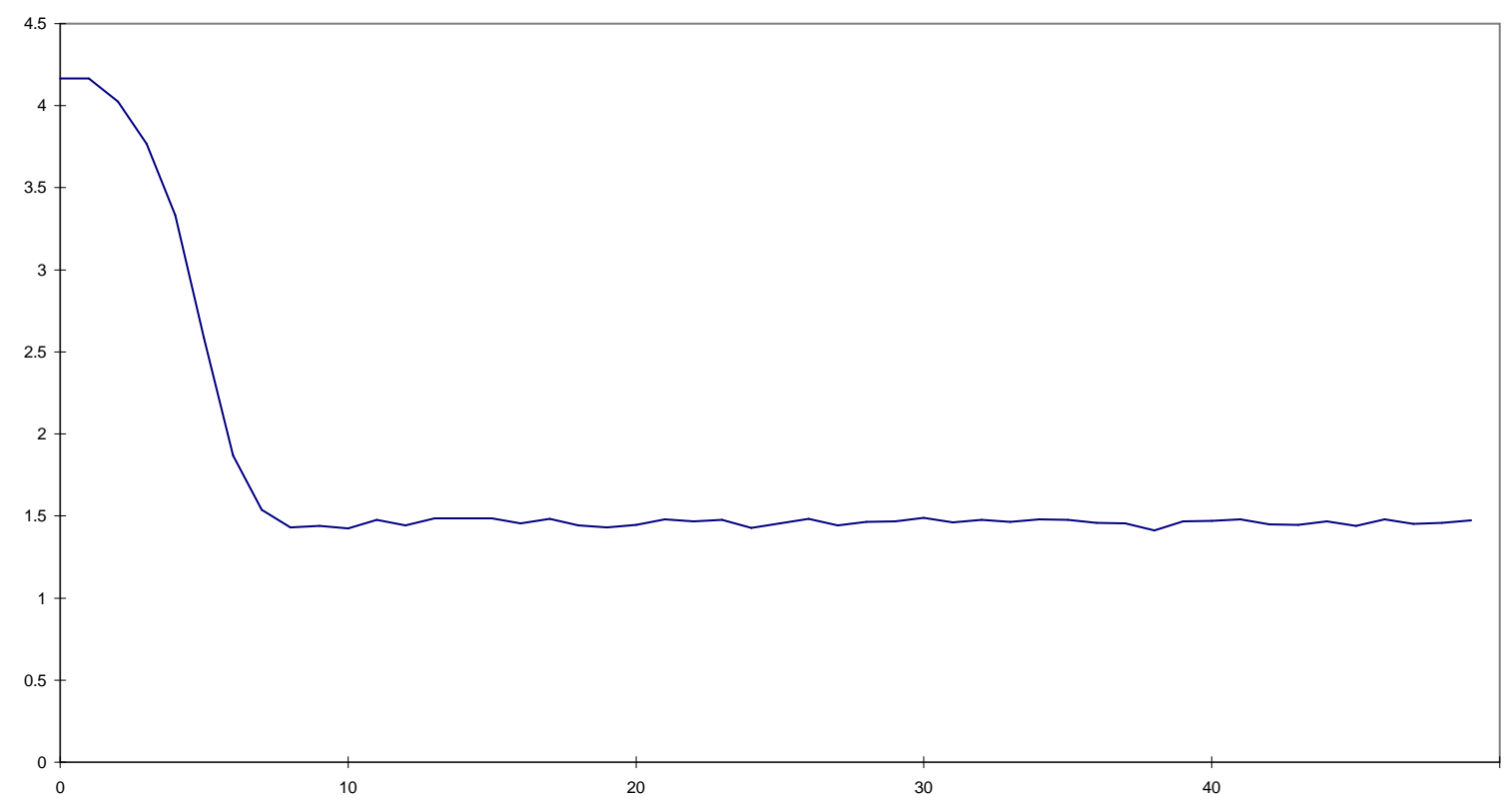

Figure 1: Price $h\left(Q_{t}\right)$ for $0 \leq t \leq 50$.

Underlying that apparent stability of aggregate supply, one observes however a more messy micro structure with a fringe of productive capacity provided by the least efficient firms (Figure 2), which keep entering and quickly die. Note also that while the total capacity of firms that manufacture stabilizes rather fast, this is not so for the total number of firms (cf. Figure 3). That is to say, for a rather long "transitional dynamic" the "carrying capacity" of the market is not saturated and the relative aggregate stability of supply and price is supported by a net inflow of firms. In a sense, during the "transitional dynamic", market selection operates less effectively allowing relatively long survival time

\footnotetext{
${ }^{6}$ The simulation used a program from the Laboratory for Simulation Development (LSD), a package providing a user friendly environment for implementation of simulation models developed by M. Valente at the International Institute for Applied Systems Analysis (IIASA). It is publicly available via Internet.
} 
also for less efficient firms and persistent "early moves advantages".

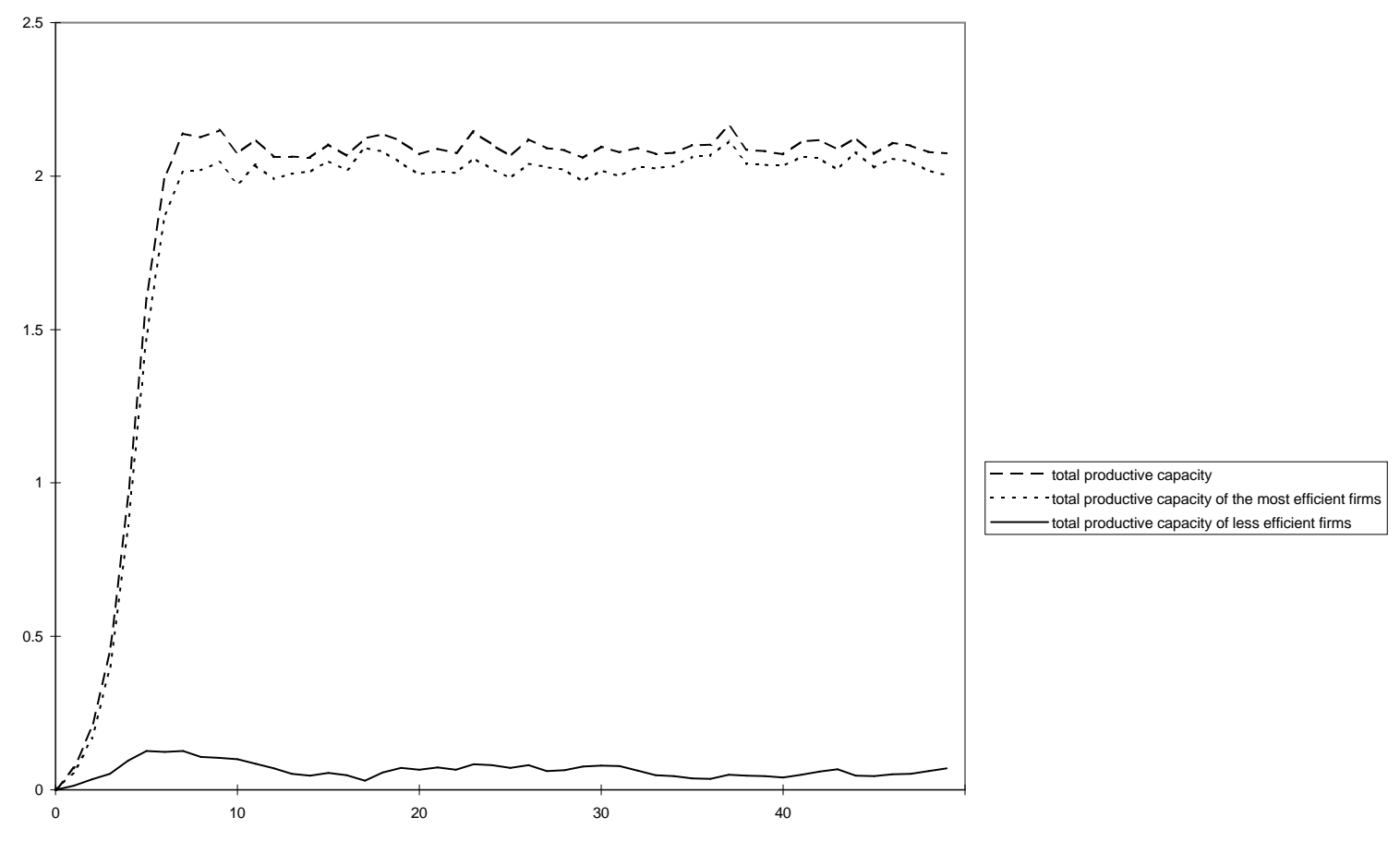

Figure 2: Productive capacities $Q_{t}, Q_{t}^{A_{1}}$ and $Q_{t}^{A_{2}}$ for $0 \leq t \leq 50$.

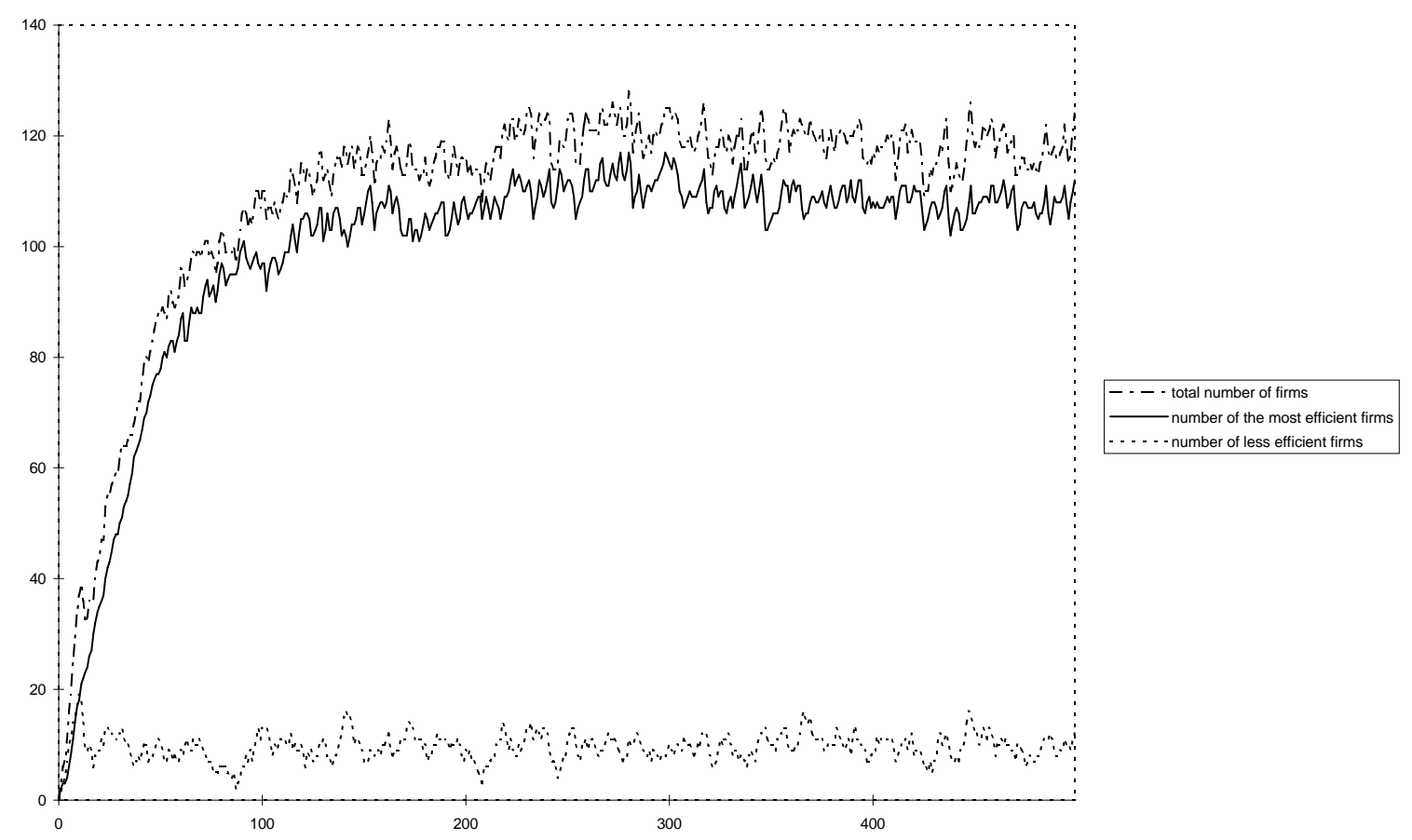

Figure 3: Numbers of firms in business for $0 \leq t \leq 500$.

Skewed size distributions appear from early on and remain thereafter (Figures 4 and 5), although - given the very simplified assumptions of our model (including the absence 


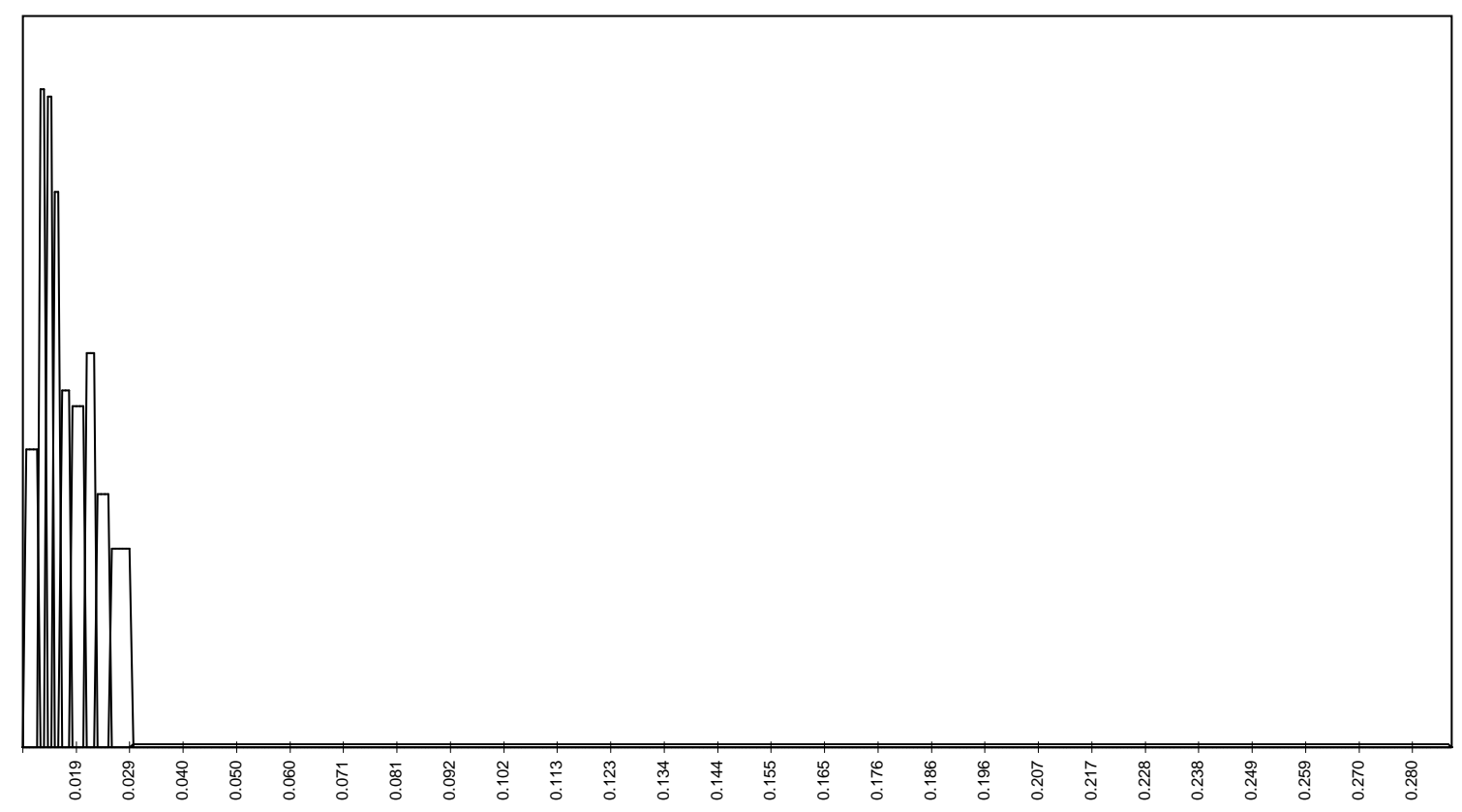

Figure 4: Size distribution of firms for $t=50$.

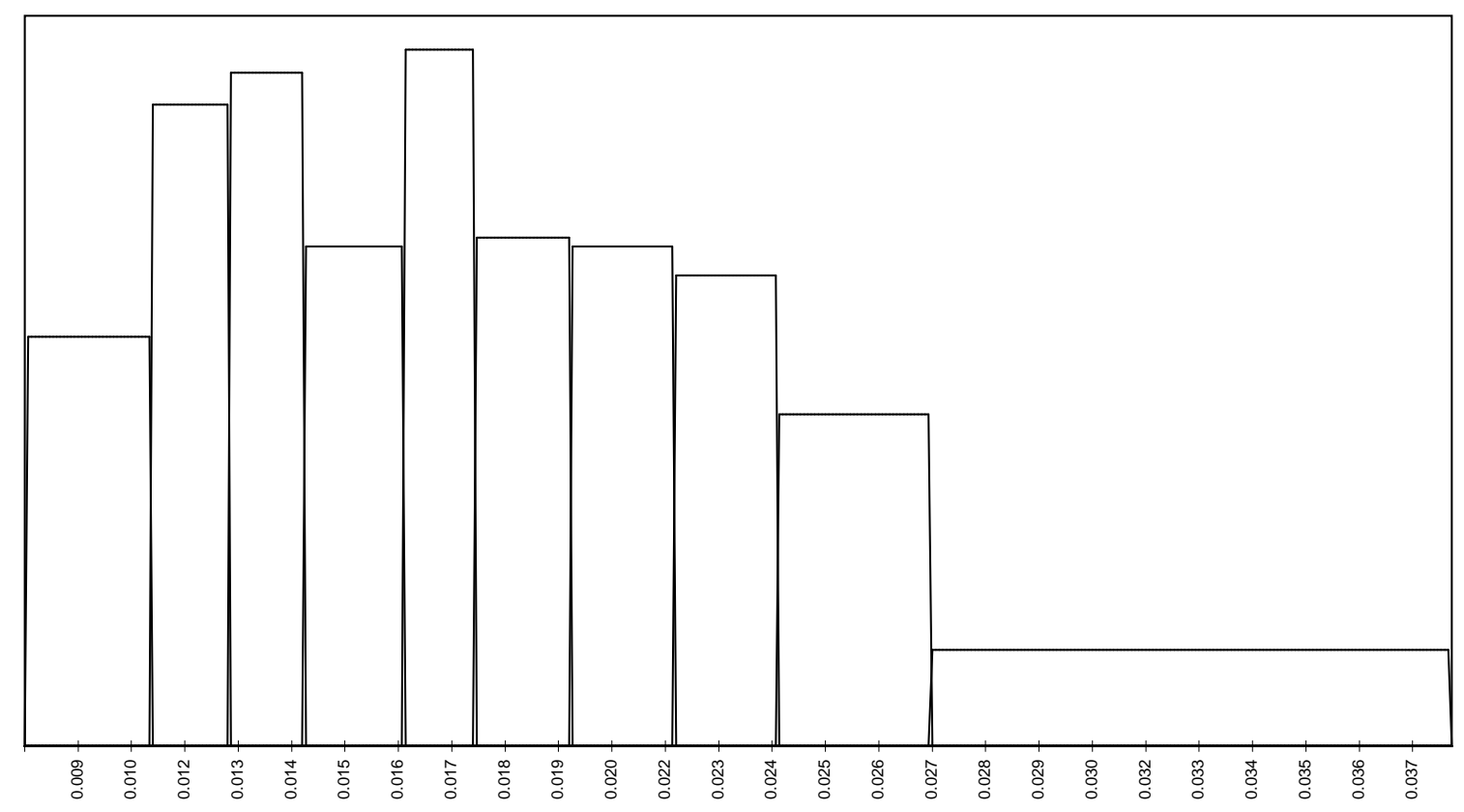

Figure 5: Size distribution of firms for $t=500$.

of learning by incumbents) - long-term skewness falls as compared to the "transitional" phase. When both production capacity and number of firms approach their stationary regimes, the competitive presure prevents all firms (including the most efficient ones) from expanding indefinitely. Relatedly, the ensuing age distribution (cf. Figures 6 and 7) assures that even the most efficient firms are bound to disappear in the long term. Indeed, 


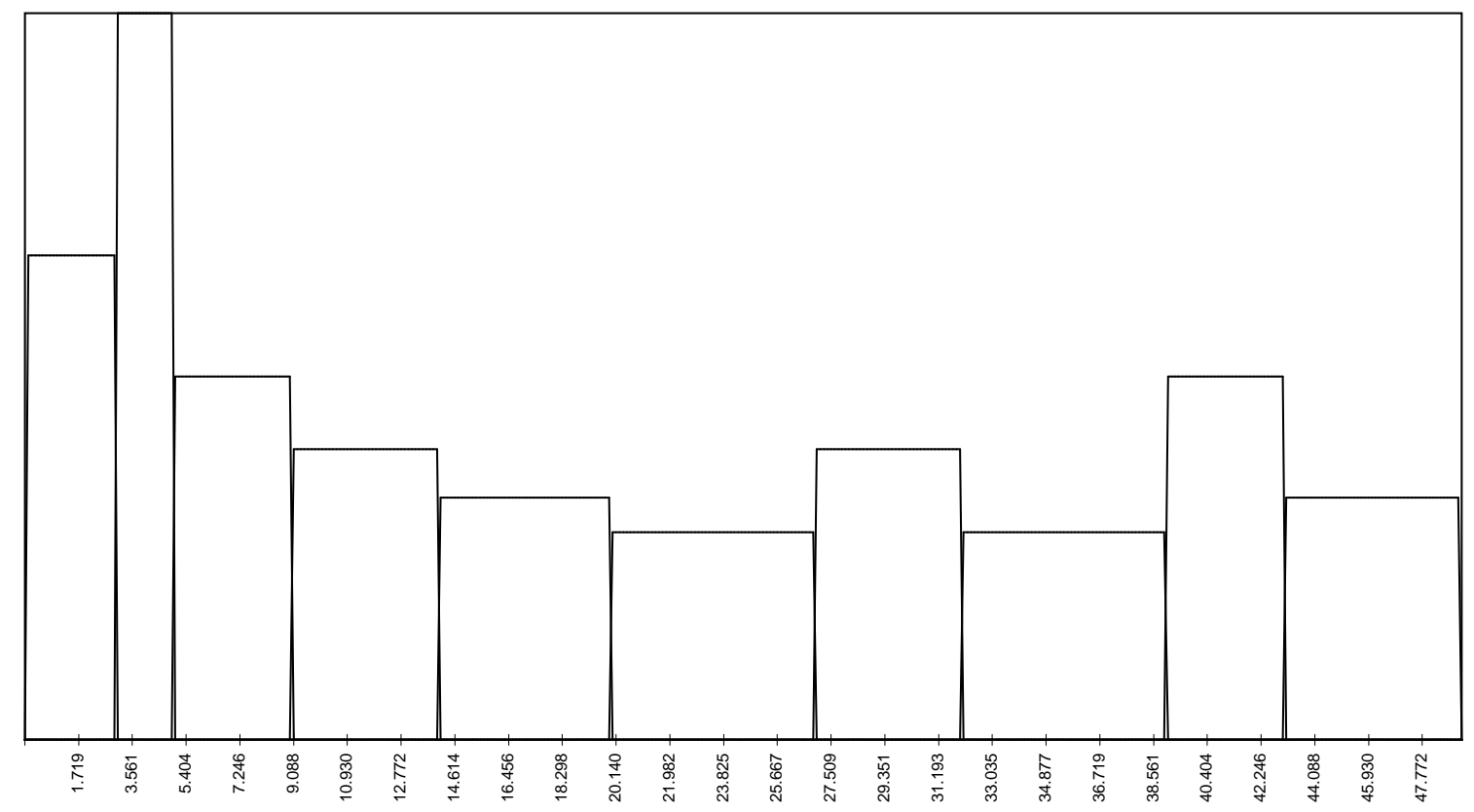

Figure 6: Age distribution of firms for $t=50$.

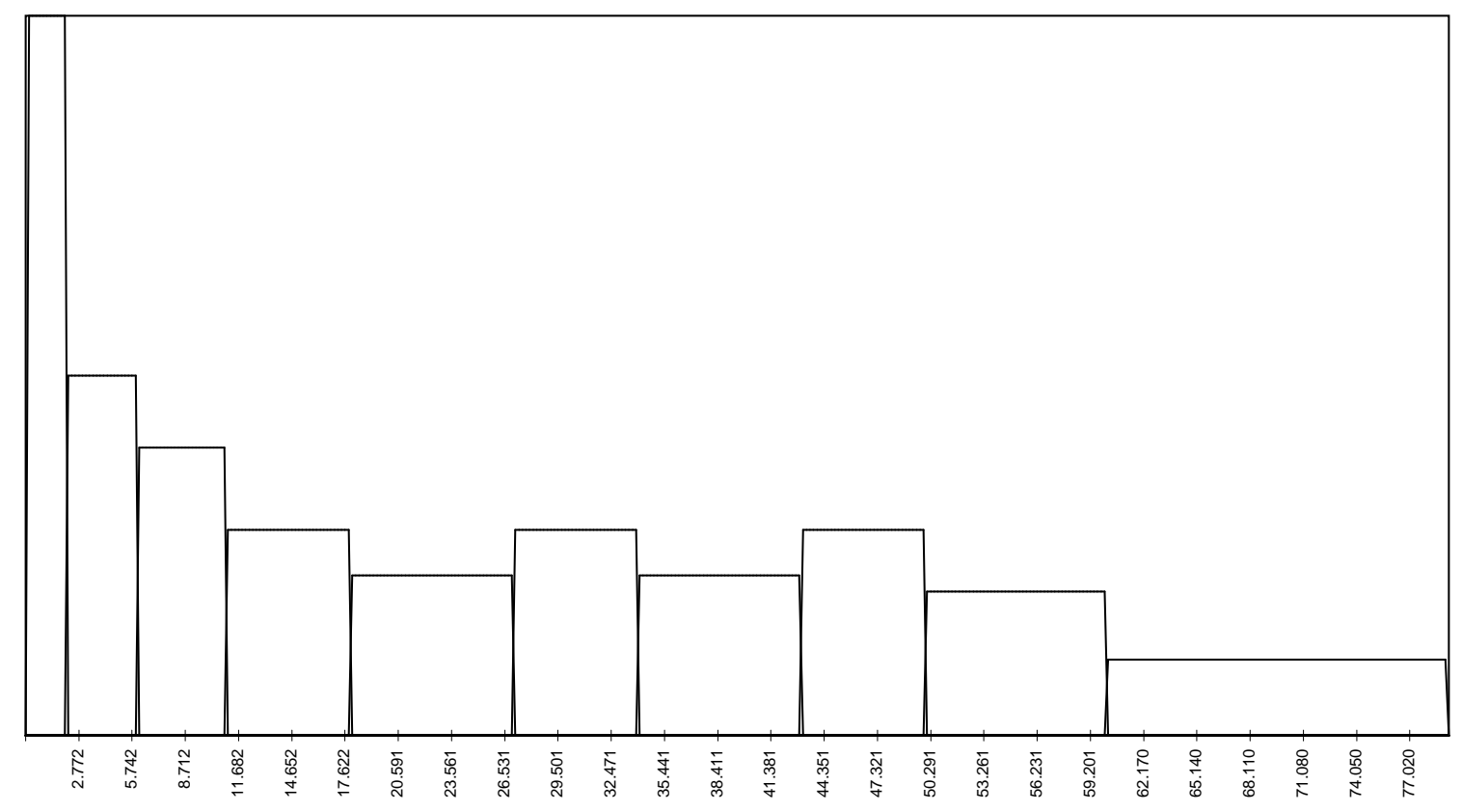

Figure 7: Age distribution of firms for $t=500$.

the picture is very similar to the Marshallian view of the "forest", mentioned earlier, with a persistent turnover of trees (with, of course, the marginal fringe having the highest turnover rates).

Note also that the foregoing qualitative properties hold across different parametrizations of the model, although long-term averages and the length of the "transitional dy- 
namics" depend of course on the parametrization itself.

\section{Generalizations of the Model}

Let us consider some possible generalizations of the basic model presented above, which can be studied by essentially the same analytic means, without affecting the major qualitative conclusions.

1. On purpose, in the foregoing exercise we have kept the behavioral assumptions to a minimum. In that vein, we have ruled out also any feedback from profitability to investment rules. However the qualitative properties of the model would not be affected if one allowed rate of investment to fall when the price gets close to variable costs per unit of output. In that case one would just set gross investment per unit of output at $t$ as

$$
\lambda \max \left[h\left(Q_{t}\right)-m, 0\right] \quad \text { if } h\left(Q_{t}\right)-m>\delta
$$

and

$$
(\lambda-\eta) \max \left[h\left(Q_{t}\right)-m, 0\right] \text { if } h\left(Q_{t}\right)-m \leq \delta,
$$

where $\lambda \in(0,1], \eta \in[0, \lambda)$. Here $\delta$ gives a threshold of profitability when the investment policy changes.

2. The death criterion could be dependent on the total productive capacity at time $t$. Namely, the $i$-th firm is dead at time $t$ and does not participate in the evolution of the market from $t$ on if its productive capacity $Q_{t}^{i}$ at $t$ is less than $\epsilon Q_{t}$. Here $\epsilon \in(0,1)$ is some threshold value.

3. One can allow also variable costs to vary across firms. Assume that there are $n>1$ possible levels of variable costs per unit of outputt $m_{j}, j=1,2, \ldots, n$. Allowing as above multiple entrants, we postulate that at time $t \geq 0$ the number of newcoming firms which have $A_{i}$ as output/capital ratio and $m_{j}$ as variable costs is given by $\Gamma_{n(i-1)+j}^{t}$ the $n(i-1)+j$-th coordinate of $\boldsymbol{\Gamma}^{t}$. Here $\boldsymbol{\Gamma}^{t}, t \geq 0$, are independent realizations of a $n \times k$ dimensional vector $\boldsymbol{\Gamma}$ whose coordinates are independent realizations of the random variable $\gamma$ defined in Section 2. Initial capitals of these firms are given by $\Gamma_{n(i-1)+j}$ independent realizations of the random variable $\theta$. Moreover, initial capitals for different time instants are independent and they do not depend upon the number of newcoming firms.

4. Initial capitals of newcoming firms may depend on their capital/output ratios and their variable costs. Thus, instead of a single random variable $\theta$, we may consider a 
collection of them, $\theta_{i, j}, 1 \leq i \leq k, 1 \leq j \leq n$. (Each of these variables is assumed to have a bounded support).

5. One can make $p_{0}$ the probability of "not to enter" dependent upon how close the current price is to the variable costs of the firm which is deciding whether to enter or not. Let $\phi_{j}(\cdot)$ be a decreasing function mapping $[0, \infty)$ to $\left[0, \beta_{j}\right], \beta_{j} \in(0,1], j=1,2, \ldots, n$. For example, $\phi_{j}(x)=\exp \left(-\phi_{j} x\right), \phi_{j}>0$. Then the distribution of the random variable $\gamma_{i, j}^{t}\left(Q_{t}\right)$ governing the number of firms with the output/capital ratio $A_{i}$ and the variable $\operatorname{costs} m_{j}$ that enter the market at $t$ can be as follows

$$
\gamma_{i, j}^{t}(x)= \begin{cases}0 & \text { with probability } p_{0}^{(i)} \phi_{j}\left(\max \left[h(x)-m_{j}, 0\right]\right), \\ s \quad \text { with probability } 1-\frac{p_{s}^{(i)} p_{0}^{(i)}}{1-p_{0}^{(i)}}\left\{1-\phi_{j}\left(\max \left[h(x)-m_{j}, 0\right]\right)\right\},\end{cases}
$$

where $0 \leq p_{0}^{(i)}<1, p_{s}^{(i)} \geq 0,1 \leq s \leq l^{(i)}-1, p_{l^{(i)}}^{(i)}>0, \sum_{s=0}^{l^{(i)}} p_{s}^{(i)}=1$. By $l^{(i)}$ we designate the maximum feasible number of entrants with $A_{i}$ as the capital per unit of output, hence $p_{l(i)}^{(i)}>0$. The random variables $\gamma_{i, j}^{t}(\cdot)$ are assumed to be stochastically independent in each of the indexes.

\section{Conclusions}

In this paper we have developed a formal analytical apparatus able to treat the dynamics of industrial evolution and derive some generic properties of the underlying competition process. The continuing entry flow produces a continuing turnover in the firm population of the sort observed in real data. The size distribution of firms emerges as a derivative consequence of the combination of heterogeneity and turnover. Although "snapshots" of the distribution at different points in time might be similar (after the industry has approached its long term dynamic path), the firms occupying particular places in the distribution are generally different. This does not only apply to the lower end of the distribution, but to the whole universe of firms: in fact, we proved, under quite general conditions, that all firms are bound to die in a finite random time with probability one.

Moreover, the long-run proportions of firms of different efficiency levels reflect the interplay of selection forces and entry rates in a fashion roughly analogous to the analysis of gene frequencies provided by the Hardy - Weinberg laws of population biology ${ }^{7}$.

\footnotetext{
${ }^{7}$ The Hardy - Weinberg laws provide a quantitative statement of the fact that "deleterious" genes are continuously eliminated from the population by natural selection forces, but are replenished by mutation (see Wilson and Bossert (1971)).
} 
The view of the outcomes of the competition process, in a sense, is a formal vindication of the intuition of classical economists that conditions of entry and (heterogeneous) techniques of production determine some sort of "centers of gravity" around which actual prices, quantities and profitabilities persistently fluctuate. Indeed, on the ground of the foregoing model, one is able to establish the limit properties of those time averages.

As mentioned earlier, the model is suitable to several extensions. An obvious one is the account of an endogenous process of arrival of new techniques and, hence, new productivity levels.

\section{References}

Afuah, A. N., and Utterback, J. M. (1997). "Responding to Structural Industry Changes: A Technological Evolution Perspective," Industrial and Corporate Change, forthcoming.

Baldwin, J. R. (1995). The Dynamics of Industrial Competition. Cambridge: Cambridge University Press.

Carroll, J. R. (1997). "Long-term Evolutionary Changes in Organizational Populations: Theory, Models and Empirical Findings," Industrial and Corporate Change, forthcoming.

Carroll, J. R., and Hannan, M. T. (eds.) (1995). Organizations in Industry. New York and Oxford: Oxford University Press.

Doob, J. L. (1953). Stochastic Processes. New York: John Wiley.

Dosi, G., and Lovallo, D. (1997). "Rational Enterpreneurs or Optimistic Martyrs? Some Considerations on Technological Regimes, Corporate Entries and the Evolutionary Role of Decision Biases," in R. Garud, P. Nayyar and Z. Shapiro (eds.) Technological Foresights and Oversights. New York and Cambridge: Cambridge University Press, forthcoming.

Dosi, G., Malerba, F., Marsili, O., and Orsenigo, L. (1997). "Industrial Structure and Dynamics: Evidence, Interpretations and Puzzles," Industrial and Corporate Change, forthcoming. 
Dosi, G., Marsili, O., Orsenigo, L., and Salvatore, R. (1995). "Learning, Market Selection and the Evolution of Industrial Structures," Small Business Economics 7, 411-436.

Dunne, T., Roberts, M. J., and Samuelson, L. (1988). "Patterns of Firm Entry and Exit in U.S. Manufacturing Industries," Rand Journal of Economics 19, 495-515.

Ericson, R., and Pakes, A. (1995). "Markov-Perfect Industry Dynamics: A Framework for Empirical Work," Review of Economic Studies 62, 53-82.

Hannan, M. T., and Freeman, J. (1989). Organizational Ecology. Cambridge, Mass.: Harvard University Press.

Hopenheyn, H. A. (1992). "Entry, Exit and Firm Dynamics in Long Run Equilibrium," Econometrica 60, 1127-1150.

Jensen, J. B., and McGuckin, R. H. (1997). "Firm Performance and Evolution: Empirical Regularities in Microdata," Industrial and Corporate Change, forthcoming.

Jovanovic, B. (1982). "Selection and Evolution of Industry," Econometrica 50, 649-670.

Klepper, S. (1997). "Industry Life Cycles," Industrial and Corporate Change, forthcoming.

Nelson, R. R., and Winter, S. G. (1982). An Evolutionary Theory of Economic Change. Cambridge, Mass.: The Belknap Press of Harvard University Press.

Scherer, F. M., and Ross, D. (1990). Industrial Market Structure and Economic Performance. Boston: H. Mifflin.

Wilson, E. O., and Bossert, W. H. (1971). A Primer of Population Biology. Sunderland, Mass.: Sinauer. 